\title{
Binocular Information Acquisition and Visual Memory
}

\author{
Thomas A. Busey \\ Indiana University Bloomington
}

\author{
Geoffrey R. Loftus \\ University of Washington
}

\begin{abstract}
Mechanisms underlying the binocular combination of visual information were investigated within the context of a visual information acquisition theory proposed by Loftus, Busey, and their colleagues (e.g., as described by T. A. Busey \& G. R. Loftus, 1994). A central assumption of the theory is that of a sensory threshold, which engenders an information loss such that information processing subsequent to the threshold is assumed to occur only when the magnitude of a sensory representation triggered by the stimulus exceeds the threshold. The presumed sensory threshold may be situated prior to or subsequent to the point at which the information from the two eyes combines. The location of this threshold was investigated in 3 experiments that provided conclusions about the location of the sensory thresholds and the mechanisms of binocular combination. It is concluded that a linear summation mechanism, an independent sampling information acquisition model, and both pre- and postcombinatorial sources of information loss are required to account for the data.
\end{abstract}

Visual information acquisition begins at the perceptual level with photons arriving at each of an observer's two eyes. The information contained in the pattern of arriving photons may represent something as simple as a monochromatic patch of light or as complicated as a naturalistic scene. Regardless of the stimulus, however, the geometry of binocular vision and the separation between the two eyes generally dictate that slightly different versions of this information arrive at each eye.

Despite initially acquiring two separate representations of the visual scene, it is only under rare circumstances that observers report seeing more than one view of the world. This plus other evidence from the stereo depth perception literature (e.g., Julesz, 1971) and the binocular combination literature (e.g., Blake \& Fox, 1973; Blake, Sloane, \& Fox, 1981) implies that the visual system must combine the outputs of the two eyes into a single unitary sensory representation and then, on the basis of this representation, proceed with further information-processing mechanisms to acquire task-relevant information.

In this article, we report research on binocular coding investigated within the context of a visual information acquisition theory described by the two of us and our colleagues (Busey \& Loftus, 1994; Loftus, Busey, \& Senders, 1993; Loftus \& Ruthruff, 1994). The theory consists of a

Thomas A. Busey, Department of Psychology, Indiana University Bloomington; Geoffrey R. Loftus, Department of Psychology, University of Washington.

This research was supported by National Institute of Mental Health (NIMH) Predoctoral Fellowship MH10367-02 and by NIMH Grant MH 41637.

We would like to thank George Wolford for many helpful comments concerning the interpretation and modeling of these data. Helpful comments by Randy Blake and an anonymous reviewer were also appreciated.

Correspondence concerning this article should be addressed to Thomas A. Busey, Department of Psychology, Indiana University, Bloomington, Indiana 47405. Electronic mail may be sent to busey@indiana.edu. sensory front end followed by information acquisition components. The sensory front end is responsible for generating the sensory representation indicated above, and subsequent information acquisition mechanisms are responsible for acquiring task-relevant information. This theory successfully predicts performance in a number of different information-processing paradigms including digit recall (Busey \& Loftus, 1994; Loftus et al., 1993; Loftus, Duncan, \& Gehrig, 1992; Loftus \& Ruthruff, 1994), picture recognition (Loftus \& McLean, 1998), and synchrony judgment and partial report (Loftus \& Irwin, in press). Despite these successes, however, the current conceptualization of the theory does not specify how information from the two eyes combines to create a unitary sensory stimulus representation.

We have two goals in this article. Our first goal is to account for the mechanisms that combine information from the two eyes in information-processing tasks. Our second goal is to examine the sources of information loss that result from a sensory nonlinearity that proved essential to account for past data. We demonstrate that these two goals cannot be addressed separately but are intimately related. Below we propose an extension of the Loftus and Busey theory and focus on a crucial component of it termed a sensory threshold, which entails a specific source of information loss in the information-processing pathway. We address two central questions: First, is the sensory threshold prior or subsequent to this point at which information from the two eyes combines? Second, what mechanisms are responsible for combining the two sources of information into a single unitary representation? ${ }^{1}$

\footnotetext{
${ }^{1}$ The range of binocular phenomena is large, and we confine the domain of our theory building to relatively low-contrast stimuli. The majority of binocular summation experiments have been conducted with low-contrast stimuli presented for brief durations in detection tasks, and these findings provide a good database from which combinatorial theories can be developed.
} 


\section{Mechanisms of Binocular Combination}

The major empirical question guiding binocular summation experiments has been How much better are two eyes than one eye? The answer has not been simple. As detailed in excellent reviews by Blake and Fox (1973; Blake et al., 1981), binocular performance may exceed monocular performance by various magnitudes. Binocular performance above monocular performance can be expected on statistical grounds, because a binocular stimulus provides two opportunities to detect a near-threshold stimulus. Blake and Fox (1973) formalized this into the probability summation prediction, which is implied by the assumption that the two opportunities were made independently. This model assumes an or rule for combination of the information from both eyes, such that the stimulus is detected if either the left or the right eye detects it. Other models of binocular combination can be produced through consideration of the neural mechanisms that combine the signals from the two eyes, which include superadditive summation (interaction), additive (linear) summation, and subadditive summation (inhibition). Interaction implies that the output of a system combining the inputs from the two eyes exceeds the sum of the inputs. Linear summation implies that the output is the sum of the inputs, and subadditive summation implies that the output is less than the sum of the inputs. The probability summation prediction has become somewhat of a measuring stick from which conclusions about the combinatorial mechanisms can be derived. Blake and Fox (1973) concluded that if binocular performance exceeds a probability summation prediction based on monocular performance, this implies genuine neural interaction (i.e., an additive or superadditive summation mechanism). They argued that the probability summation model accordingly provides a baseline to detect combinatorial mechanisms that imply more interaction than the or rule described above.

Although the focus of binocular combination experiments has been on the nature of the combination mechanisms (i.e., neural interaction, linear summation, or inhibition), we demonstrate that such questions cannot be addressed without consideration of both pre- and postcombinatorial sources of information loss. In particular, we argue that a postcombinatorial threshold mechanism must play a major role when binocular combination data are used to infer the mechanisms of combination.

\section{Binocular Superiority Depends on Temporal Overlap}

The question of the combinatorial mechanisms subserving binocular combination is best addressed by varying the onsets of the presentations to each eye. For example, consider the probability summation model, which assumes that each eye provides an independent chance to detect a near-threshold stimulus. If this model holds, performance will remain constant if the two eyes are stimulated simultaneously or if first one eye and then the other eye is stimulated. Conversely, improved binocular performance with simultaneous presentation compared with successive presentation would constitute evidence against the probabil- ity summation model. Thus the temporal overlap of the two presentations in a binocular condition provides a strong test of binocular summation models, and below we review relevant temporal overlap experiments.

Three studies of binocular combination demonstrate the utility of varying the temporal onsets of a binocular stimulus to each eye, and all three sets of authors have argued for neural interactions (predictions above those of probability summation) between the two eyes to produce binocular performance. Westendorf, Blake, and Fox (1972) presented contrast increments to both eyes in a detection task. The stimulus onset asynchrony (SOA) between the two presentations was either $0 \mathrm{~ms}$ or $100 \mathrm{~ms}$. Westendorf et al. found binocular performance above that predicted from probability summation for the simultaneous presentations but binocular performance consistent with probability summation for the 100-ms SOA. They concluded that binocular performance cannot be predicted solely from probabilistic considerations but that the superiority at the 0 -ms SOA resulted from neural interaction between the two eyes.

An earlier study by Matin (1962) demonstrated the utility of varying the temporal overiap of the two presentations. Matin varied the interval separating the two presentations over a wide range and found that SOAs less than $100 \mathrm{~ms}$ produced binocular performance that was superior to that predicted by probability summation. He concluded that the two eyes were not independent detectors but that detection instead resulted from neural summation between the two eyes.

Although much of the work on binocular summation has relied on detection tasks, Eriksen and his colleagues (Eriksen \& Greenspon, 1968; Eriksen, Greenspon, Lappin, \& Carlson, 1966) examined letter identification in a threealternative forced-choice procedure. As with previous findings, they found that for SOAs shorter than $50 \mathrm{~ms}$, binocular performance exceeded that expected from the probability summation prediction.

\section{Existing Models of Binocular Combination}

In short, the available evidence disconfirms a probability summation mechanism and has led investigators to posit neural interaction as the combination mechanism mediating binocular combination. However, these data also clearly indicate that no single combinatorial mechanism can account for binocular summation at all SOAs, because binocular performance changes with SOA. Thus a complete description must account for the amount of temporal overlap that is produced by a nonzero SOA binocular stimulus. The present article represents such an approach, although it is motivated, in part, by extant binocular summation models. Two of the most influential are the binocular energy detector model of Legge (1984a, 1984b) and the interocular suppression model of Cogan and colleagues (Cogan, 1987; Cogan, Clarke, Chan, \& Rossi, 1991).

The binocular energy detector model extends the signal detection model of Green and Swets (1974) to binocular summation. The model consists of two monocular channels that are subjected to input noise before undergoing a 
squaring function. The results are linearly summed, and the final binocular output results from a compressive nonlinearity and the addition of central noise. This model predicts a quadratic summation relationship between binocular and monocular thresholds, which results from the fact that when two noisy signals are added, the standard deviation increases by the square root of the number of signals. Although this model can account for a diverse range of low-contrast phenomena, all of the binocular stimuli used to test the model consist of simultaneous presentations to both eyes. The model does not include a temporal representation of the physical stimulus, and thus it cannot be used to account for nonzero SOA binocular presentations. As described above, these stimuli provide strong tests of binocular summation models because they can be used to determine how the range of binocular summation varies with the onsets of the two presentations of a binocular stimulus.

Alexander Cogan (Cogan, 1987; Cogan et al., 1991) presented a model that can qualitatively account for the effects of varying the SOA between the two presentations of a binocular stimulus. We describe this model and fit it to our data in the General Discussion section. In brief, it is as follows. The combination mechanism is relatively complex: Information registering in either eye both provides excitatory input to the monocular channel and inhibits the other monocular channel. In a separate mechanism, the information from the two eyes combines into a binocular fused channel, and the final binocular output is the sum of the combined monocular channels and the binocular fused channel. This model accounts for increments and decrements in binocular and monocular presentations and will also qualitatively account for the effects of nonzero SOAs in binocular presentations. However, Cogan did not provide quantitative fits to variable-SOA data. The importance of quantitative fits becomes apparent in the General Discussion section of the current article, but to anticipate, two models that share similar theoretical structures and thus provide qualitatively identical predictions do not give the same quantitative predictions. Indeed, we test and disconfirm Cogan's model on the basis of poor quantitative fits.

One conclusion is clear from the existing binocular summation data and theories: A complete account of binocular summation must include both descriptions of how information from the two eyes combines as well as how this relationship changes as the SOA between the two presentations is varied. This, along with a description of the sources of information loss that contribute to performance, is the major theoretical contribution of the current work.

The remainder of this article is organized as follows. First, we explicitly describe the task that we are trying to model. Second, we briefly outline our theory and propose two (not mutually exclusive) candidate extensions to it that may account for performance in tasks designed expressly to investigate binocular combination. Third, we describe data from three experiments designed to evaluate the two extensions of the theory and, more generally, to reveal the nature of the information combination mechanisms. Fourth, we conclude that neither extension alone will completely account for all of the observed characteristics of the data, although a combination of the two extensions does provide a good account of the data. Fifth, we make specific conclusions about the nature of the information combination mechanisms and, on the basis of these conclusions, describe theoretical implications for researchers studying the nature of the combinatorial mechanisms. Finally, we apply Cogan's model to our data to examine how the choice of combination mechanisms affects our conclusions. We argue that the question of the nature of the combinatorial mechanism is intimately tied to the question of the location of the sensory threshold and that binocular performance above that predicted by probability summation need not imply neural interactions between the two monocular channels.

\section{An Information-Processing Task}

The task that we attempt to model is complex enough to provide generalizability to everyday tasks but simple enough to be plausibly described with a relatively simple and concise theory. In this task, four digits are presented to an observer whose job is to report as many of them as possible, in their correct order, and to guess if necessary. The basic performance measure, $p$, is the proportion of correctly reported digits, in their correct locations, adjusted for the guessing probability of $0.10 .^{2}$

\section{Theory}

We (Busey \& Loftus, 1994) described a theory, which we termed the LST (linear systems theory) model, that has successfully accounted for performance in this digit-recall task. ${ }^{3}$ In this section we summarize the theory, and in the next section we develop two extensions of it that are designed to account for the combinatorial mechanisms involved in binocularly viewed stimuli. This theory conjoins a linear-filter front end, common in the vision literature, with an independent information-sampling model that operates on the output of the linear-filter front end to acquire task-relevant information. Figure 1 presents three functions that are relevant throughout our discussion of the theory. Note also that complete equations for all models can be found in Appendix $A$ and that the units and dependencies of all model parameters and functions can be found in a glossary in Appendix B.

\section{Linear-Filter Front End}

The initial response in the visual system to a briefly presented stimulus can be modeled as the output of a band-pass or low-pass linear filter (e.g., Sperling \& Melchner, 1978; Watson, 1986). Rapid temporal changes in the stimulus are temporally blurred by a visual system that cannot keep up with the stimulus; slower temporal changes are better represented. The exact nature of the response can

\footnotetext{
${ }^{2}$ The guessing formula is $p=(x-0.1) / 0.9$, where $x$ is the raw proportion correct and $p$ is the corrected proportion.

${ }^{3}$ See also Loftus, Busey, and Senders (1993) and Loftus and Ruthruff (1994).
} 

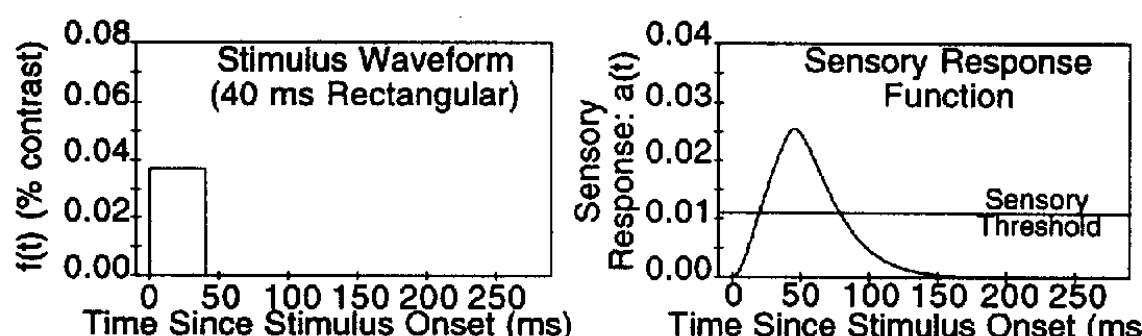

Time Since Stimulus Onset (ms)

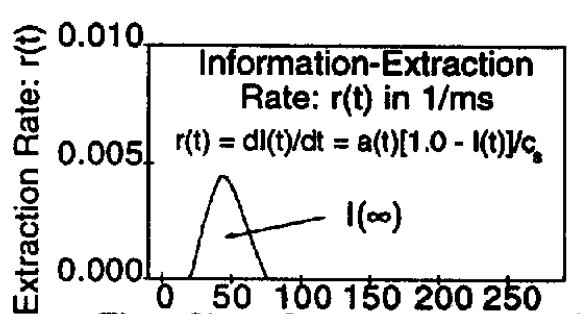

Time Since Stimulus Onset (ms)

Figure 1. Theoretical components of the linear-filter model of character identification. Left panel: A stimulus is characterized by its changes in contrast over time. Middle panel: The stimulus engenders a response in the visual system that is a function of the stimulus input function $f(t)$ and the impulse response function $g(t)$. A sensory threshold is assessed, such that further information processing does not proceed unless the sensory response exceeds the sensory threshold. Right panel: If the sensory response exceeds the threshold, further information processing takes place at a rate defined by $r(t)$. This rate is proportional to the product of the above-threshold sensory response and the proportion of remaining stimulus information. Performance in terms of the proportion of correctly recalled digits is assumed to be proportional to the area under the information-acquisition rate function (which represents total acquired information).

be derived by specifying an impulse response function for the linear filter that describes the severity of the temporal blurring or, thought of in another way, the amount of response that persists following the termination of the physical stimulus. Under low-luminance conditions such as the ones used in our experiments, the filter is usually assumed to be low-pass, which means that the visual system reproduces progressively higher temporal frequencies progressively less faithfully.

The shape of the impulse response function, $g(t)$, dictates the type of temporal blurring. In keeping with previous sensory literature (e.g., Watson, 1979, 1986), we have chosen this function to be the difference of two gamma functions, each with a different time constant,

$$
g(t)=\frac{(t / \tau)^{n-1} e^{-t / \tau}}{\tau(n-1) !}-s\left[\frac{(t / r \tau)^{n-1} e^{-t / r \tau}}{r \tau(n-1) !}\right]
$$

where $\tau$ represents the time-constant of the gamma function and provides an estimate of the temporal response properties of the mechanisms mediating a given task. The sensory response function component of the LST theory is based on work from Andrew Watson (1986), George Sperling (Sperling \& Sondhi, 1968), and others working in the temporal domain of perception. The left panel of Figure 2 shows example impulse response functions.

The first term in Equation 1 represents an excitatory component, whereas the second term represents an inhibitory component of the response, which tends to sharpen the response and allows it to respond to higher temporal frequencies. The parameter $r$ represents the ratio of the time-constant of the inhibitory component of the response to the excitatory component of the response, and $s$ represents the magnitude of the temporal inhibition component. The parameter $n$ represents the number of exponentially decaying stages in each gamma process, and for the current modeling we fix it at 9, although the fit of linear-filter models to data is relatively unaffected by different values of $n$ (Burr \& Morrone, 1996).

Identification data for letter stimuli such as those used in the present study are often modeled by setting $s$ to 0 (Busey, 1994), which gives a monotonic impulse response function $g(t)$ as shown by the solid curve in the left panel of Figure 2. An alternative way of representing the same information is by taking the Fourier transform of the impulse response function $g(t)$, which results in a temporal contrast sensitivity function (TCSF). The TCSF plot shows the sensitivity of a system to different temporal frequencies. The TCSF corresponding to the solid line in the left panel of Figure 2 is given by the solid line in the right panel. These curves represent a purely sustained response and give a monotonically decreasing TCSF, as shown in the right panel of Figure 2.

Detection data for stimuli containing mainly low spatial frequencies, or stimuli presented on bright backgrounds, often are modeled by $s>0$ (Watson, 1986). In this case the impulse response inhibits processing after an initial excitatory response, which results in an inhibitory lobe in the impulse response function $g(t)$ (shown by the dashed line in the left panel of Figure 2) and a characteristic TCSF with a decrease in sensitivity at low temporal frequencies (shown by the dashed curve in the right panel of Figure 2).

The temporal characteristics of the physical stimulus are summarized by a function describing contrast over time since stimulus onset, called the stimulus input function. This represents the physical stimulus, not the visual system's response to this stimulus. For the stimuli discussed here, the digits simply appeared at some contrast, $\Phi$, for some controlled stimulus duration, and then disappeared. Thus the stimulus input function $f(t)$ can be described as

$$
f(t)=\begin{array}{ll}
\Phi & (0 \leq t \leq d) \\
0 & \text { elsewhere }
\end{array}
$$



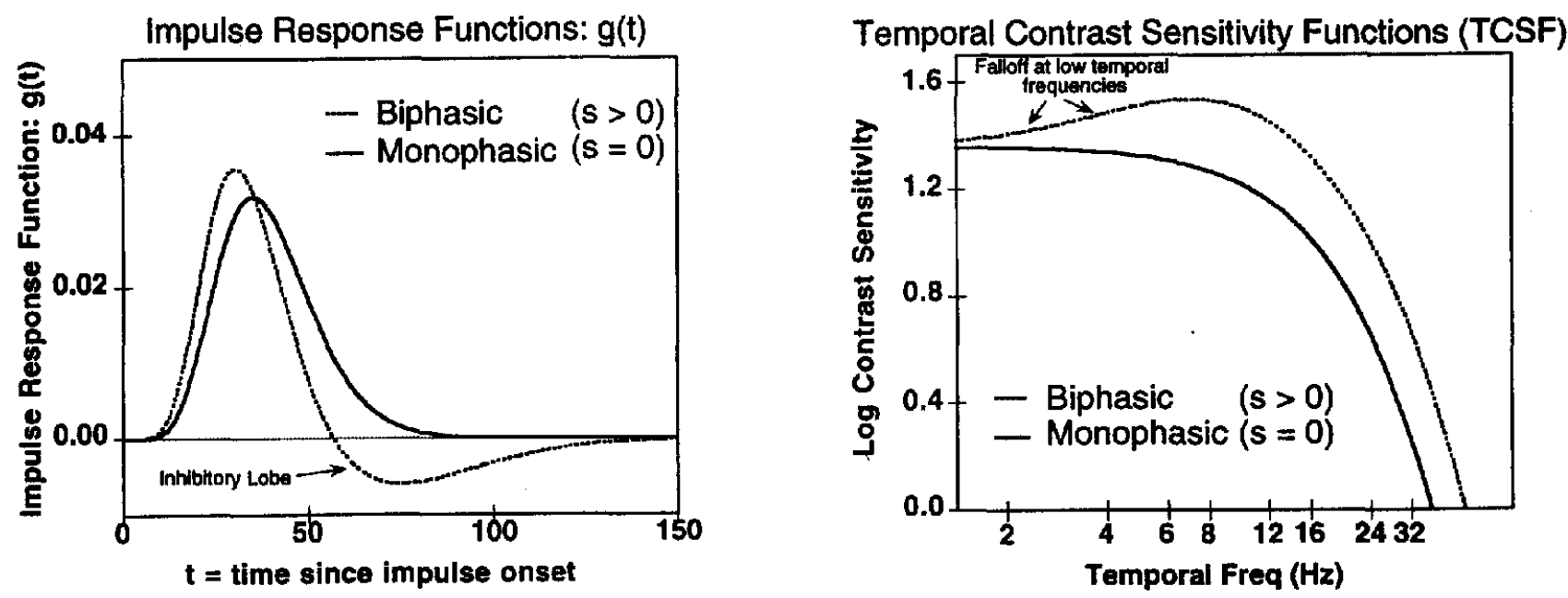

Figure 2. The temporal frequencies (freq) underlying a task may be characterized by an impulse response function (left panel), which characterizes the time course of the perceptual response engendered by a stimulus, or by the temporal contrast sensitivity function (TCSF; right panel), which characterizes the fidelity by which the pathways subserving a given task pass different temporal frequencies. The TCSF plots are the Fourier transform of the impulse response functions into frequency space. High spatial frequency stimuli tend to elicit monophasic impulse response functions, which have no falloff at low temporal frequencies in the TCSF plot. Stimuli containing low spatial frequencies tend to elicit biphasic impulse response functions that contain an inhibitory lobe. This temporal inhibition acts to sharpen the response of the visual pathway, allowing it to respond to faster changes in the visual scene. However, this inhibition causes a falloff at low temporal frequencies in the TCSF plot, which results from a tendency for the biphasic impulse response function to inhibit itself when processing slow temporal changes. Parameters used: monophasic, $n=$ $9, \tau=4.38, s=0$; biphasic, $n=9, \tau=3.58, r=2.0, s=0.39$.

where $\Phi$ represents stimulus contrast and $t$ is time since stimulus onset. ${ }^{4}$

We term the visual system's response to such a stimulus a sensory response function, denoted $a(t)$, which is computed by convolving the stimulus input function with the impulse response function:

$$
a(t)=f(t) * g(t)
$$

where * represents convolution. The sensory response function represents the output of the linear-filter stage of the theory and describes the initial representation of a stimulus in the visual system.

Further information acquisition mechanisms act on this representation to acquire task-relevant information. However, prior to becoming available for information acquisition, the sensory response function is assumed to pass through a threshold device, such that only the portion of the sensory response function that exceeds a sensory threshold is available for information acquisition. Thus we define a new function $a_{\theta}(t)$ :

$$
a_{\Theta}(t)=\begin{array}{ll}
a(t)-\Theta & (a(t)>\Theta) \\
0 & (a(t) \leq \Theta)
\end{array}
$$

where $\Theta$ is the magnitude of the sensory threshold. Informa- tion acquisition cannot begin until the sensory response, $a(t)$, exceeds the sensory threshold.

As described below, once the information acquisition process begins it proceeds at a rate that is proportional to $a_{\Theta}(t)$. Before we turn to this issue, however, some notes on the nature of the sensory nonlinearity are in order. The behavior of the sensory threshold described in Equation 4 can be mimicked by other nonlinearities, and in particular by a power function of the form

$$
a_{\beta}(t)=[a(t)]^{\beta},
$$

where $\beta$ represents the magnitude of nonlinear compression. This power function has been used by Watson (1979) in his probability summation in time model and by Legge (1984a, $1984 b$ ) in a binocular summation model with $\beta=2$. Raising the sensory response function $a(t)$ to an exponent greater than 1 has properties similar to those of a hard threshold. It tends to produce more overall area when a sensory response function is tall and thin, versus short and wide. (See Figure 2

\footnotetext{
${ }^{4}$ Because the stimuli were displayed on a computer monitor, the actual function $f(t)$ flickered at $75 \mathrm{~Hz}$, albeit at a rate faster than typically resolvable by the human eye. However, as described in Appendix C of Busey and Loftus (1994), this has little implication for our theory.
} 
in Watson, 1979, for an example of this effect, where $\left|g\left(t_{i}\right)\right|$ represents the height of the sensory response function $a(t)$. As $\beta$ increases, the psychometric function becomes steeper and approximates a hard threshold). The hard-threshold model has the same behavior, and thus the two models can mimic each other. We have fit models with both hardthreshold and power-function nonlinearities, and in general we find the two formulations equivalent. However, the threshold model gives slightly better fits to the data and is also slightly easier to demonstrate graphically. In addition, there is neurological evidence in favor of this formulation from Ohzawa and Freeman (1986), as we discuss in the General Discussion. For these reasons, as well as for consistency with previous formulations (Busey \& Loftus, 1994), we present the model predictions in terms of the threshold model. However, we provide model parameters and root mean square errors (RMSEs) for the power function model in Table 3.

\section{Acquired Information: The Information Acquisition Rate}

Once the sensory response function exceeds the sensory threshold, information acquisition can begin. At time $t$ following stimulus onset, information acquisition proceeds at an instantaneous rate, termed the information acquisition rate, denoted $r(t)$, which is proportional to the product of (1) the magnitude of above-threshold sensory response and (2) some decreasing function of already acquired information. The particular form of the information acquisition formulation is similar to independent sampling models proposed by Townsend (1981), Shibuya and Bundesen (1988), Rumelhart (1970), and Massaro (1970); namely, information is presumed to be acquired at random and with replacement. The independent sampling assumption implies that the rate of acquiring information is constant and governed by a scaling parameter $1 / c_{s}$. However, because information is sampled from the stimulus with replacement, the rate of acquiring new information is proportional to the amount of not-yet-acquired information.

More specifically, let $I(t)$ be the proportion of acquired information at time $t$. The information acquisition rate, $r(t)$, is by definition the derivative of $I(t)$ with respect to time and is given by

$$
r(t) \equiv \frac{d I}{d t}=a_{\Theta}(t)\left[\frac{1.0-I(t)}{c_{s}}\right]
$$

Equation 5 states that the rate of acquiring new stimulus information is proportional to the product of the abovethreshold sensory response and the remaining stimulus information, with a constant of proportionality $1 / c_{s}$. The units of $c_{s}$ are milliseconds, and the units of $r(t)$ are $1 / \mathrm{ms}$. Appendix B contains a glossary of all model parameters and their units.

It is simple to show (e.g., Busey \& Loftus, 1994) that with this rate function, the function relating total proportion of acquired information, denoted $I(\infty)$, to the above-threshold area under $a_{\Theta}(t)$, denoted $A_{\Theta}(\infty)$, is given by

$$
I(\infty)=1.0-e^{-A \theta(\infty) / c_{s}} .
$$

The units of $A_{\theta}(\infty)$ are milliseconds, and $I(\infty)$ has units of proportion of total information.

One additional linking hypothesis is required to make quantitative predictions, which is that $p$, the proportion of correctly recalled digits, equals the total proportion of acquired information $I(\infty)$. Given this and Equation 6, our fundamental prediction emerges:

$$
p=1.0-e^{\left.-A_{\theta}(\infty)\right) / c_{s}} .
$$

An important consequence of Equation 7 is that performance, $p$, is monotonically related to the above-threshold area under the sensory response function. Thus if some condition $i$ results in more above-threshold area, $A_{\Theta}(\infty)$, than does another condition $j$, the theory predicts that performance in condition $i$ will exceed that in condition $j$. In addition, any two conditions that produce the same abovethreshold area, $A_{\Theta}(\infty)$, are predicted to have the same performance. Although Equation 7 provides a stronger description of the area-performance relation than does monotonicity, for purposes of exposition it is convenient to think in terms of the consequences of the weaker monotonicity relation, which is that more above-threshold area implies greater performance.

At the risk of redundancy, we emphasize that these relations are a consequence of Equations 1, 3, 4, 5, and 6 and are not assumptions of the theory. It is also worthwhile to note that the prediction of Equation 7 holds for any stimulus wave form, not just the rectangular wave form given in Equation 2 and used in the present experiments.

Testing Equation 7 requires an experimental design that varies $A_{\Theta}(\infty)$, the above-threshold area under the sensory response function. We typically vary the stimulus duration within several different types of conditions, including those involving binocular, monocular, and dichoptic stimuli, in order to produce empirical data that provide a strong test of the theory. When plotted against stimulus duration, digitrecall performance in a given condition is called a performance curve.

The above model formulations do not explicitly assume the presence of noise, although, as discussed below, the sensory threshold may be thought of as representing the amount of signal lost to noise. The binocular energy model of Legge (1984a, 1984b) assumes both input and central sources of noise, although formulations by Cogan (1987) do not explicitly assume noise. Noise could be explicitly incorporated into the model without changing the formulations by assuming that the height of the sensory response function gives the probability that the signal exceeds the noise at that particular instance. This is the interpretation adopted by Watson's probability summation in time (Watson, 1979), and this makes the power-function formulation of the model similar to Legge's (1984a, 1984b) model.

Complete equations for all model formulations are provided in Appendix A. 


\section{The Role of the Sensory Threshold in Binocular Combination of Ocular Channels}

The sensory threshold described in the previous section is assumed to exist prior to the information acquisition components of the information-processing pathway. As a result, we consider it a sensory component. Because no further information processing takes place unless the sensory response exceeds threshold, the threshold can be considered a source of information loss. We now ask: Does this information loss occur before or after the information from each eye is combined into a central unitary representation?

Below we describe two models that address this question. Each model extends the previously described theory by assuming that information enters each eye and engenders in each eye an initial peripheral sensory response function. These two peripheral sensory response functions then combine centrally to provide a single central sensory response function. Information is acquired on the basis of this central sensory response function, and further processing, or a task-relevant response, is made on the basis of the amount of acquired information. We describe these two models within the context of Experiment 1, which was designed to evaluate them.

\section{Experiment 1: Is the Sensory Threshold Prior or Subsequent to Binocular Combination?}

In Experiment 1 we used monocular, binocular, and dichoptic stimuli. A monocular stimulus is presented to one eye only. A binocular stimulus is presented to both eyes simultaneously. A dichoptic stimulus consists of two temporal stimulus halves, presented successively, with a zero interstimulus interval (ISI): The stimulus is presented first to one eye and then immediately to the other eye.

As we shall see, comparison of performance curves for these three presentation types allows us to isolate the location of the presumed sensory threshold. To do so, we must extend our theory such that it takes these three presentation types into account. We first assume that information from each eye is carried by a monocular channel and that each monocular channel generates a peripheral sensory response curve. The information from the monocular channels combines to produce a central sensory response curve, upon which memory performance is based. As an extension of linearity, we further assume that the two peripheral sensory response curves sum to produce the central sensory response curve. ${ }^{5}$

Support for our assumption of a linear combination mechanism comes from single-cell recording done with simple cells in cat visual cortex by Ohzawa and Freeman (1986). Using sine-wave gratings tuned to the cell's optimal orientation, they found that the light-evoked neural responses from each eye are summed linearly to produce the output of a binocular cell. Of some relevance to our theory is that Ohzawa and Freeman also found a minority of cells that deviate from this linearity prediction, which they attributed to a threshold mechanism that occurs after the linear binocular summation. We return to this in our General Discussion section.

The linearity assumption also allows quantitative evaluation of the sources of information loss, both before and after the site of binocular combination. Such a summation mechanism was adopted by Legge (1984a, 1984b), although his additional assumptions about noise may provide for different predictions of binocular performance. A more complex combination mechanism developed by Cogan (1987) is discussed in a subsequent section.

Given the assumptions described above, we are able to ask, Is there a sensory threshold for the peripheral sensory response functions, for the central sensory response curve, or for both?

\section{Two Models}

Figure 3 illustrates these two possibilities. To illustrate them, we show the responses generated by an $80-\mathrm{ms}$ dichoptic presentation (which, of course, involves a $40-\mathrm{ms}$ presentation to one eye followed by a 40-ms presentation to the other eye). The top half of Figure 3 shows the peripheral sensory threshold model, by which a peripheral threshold, $\Theta_{p}$, is assumed on the sensory response functions generated by each monocular channel. Only the above-peripheralthreshold area from the peripheral sensory response curves combines to form the central sensory response curve. All of the central sensory response curve area contributes to performance.

The bottom half of Figure 3 shows the central sensory threshold model, by which a central threshold, $\Theta_{c}$, is assumed on the central sensory response curve. All of the peripheral-curve area contributes to the central sensory response curve, but only the above-central-threshold area contributes to performance. Both thresholds are implemented through the Equation 4 thresholding operation.

\section{Predictions: Monocular Versus Dichoptic Conditions}

Evaluating these two candidate models entails the following logic. Consider an 80-ms monocular presentation and its corresponding sensory response curve, shown in the left panel of Figure 4. This monocular presentation implies the same above-central-threshold area (1.4 in this example) for both peripheral and central sensory threshold models. The location of a sensory threshold is irrelevant for this condition because information is presented to only one eye, and there is no information to combine from the other eye. This makes the monocular condition a good comparison stimulus because monocular performance is predicted to be the same by both models.

\footnotetext{
${ }^{5}$ An alternative formulation has been suggested by Randolph Blake. Rather than two independent monocular channels, Blake suggests thinking in terms of binocularly tuned neurons that vary in their ocular dominance. Either formulation allows consideration of the question "Is the information loss (due to the sensory threshold) before or after binocular combination?"
} 


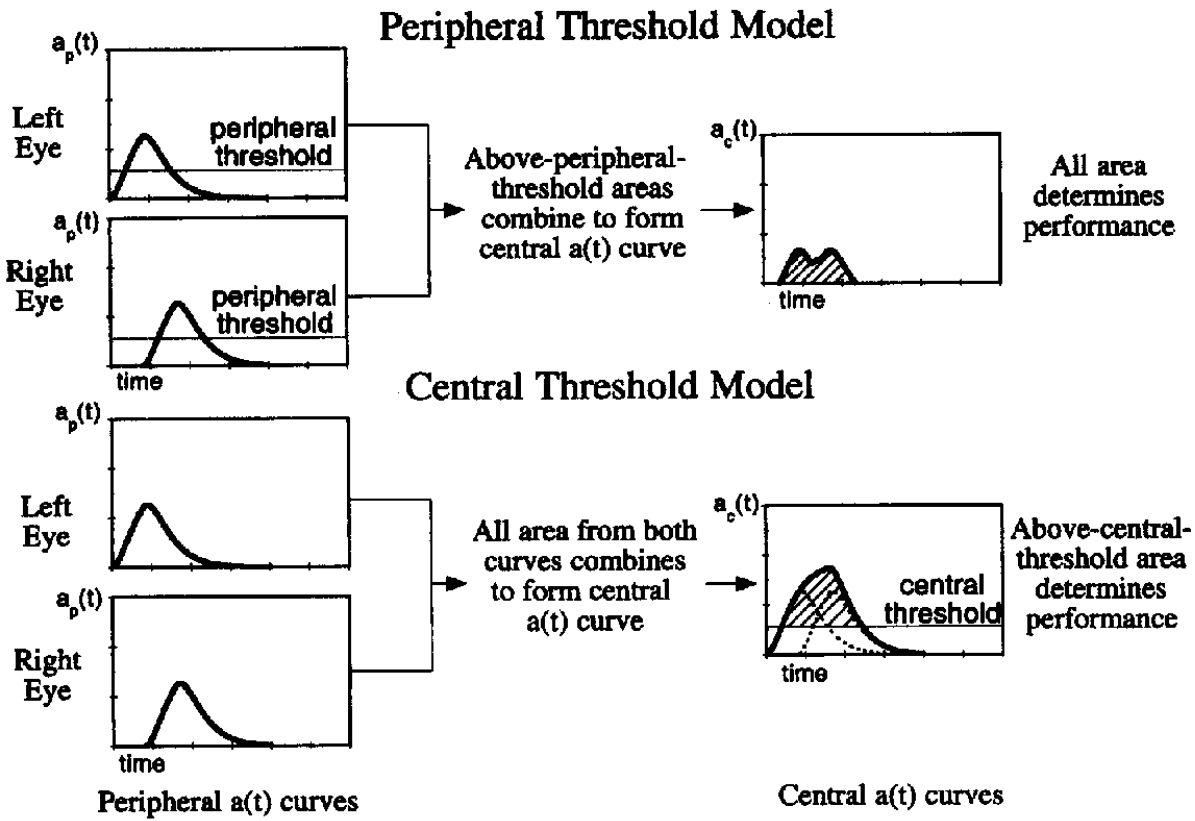

Figure 3. The peripheral threshold and central threshold models, along with the responses generated by a dichoptic presentation. Peripheral threshold model: Information enters each eye and engenders two peripheral sensory response curves, $a_{p}(t)$. These curves pass through the peripheral threshold, $\Theta_{p}$, such that only the above-peripheral-threshold area combines centrally to form the central sensory response curve, $a_{c, \Theta}(t)$. All of the area under this function determines performance. Central threshold model: The two presentations engender two peripheral sensory response curves, but under the central threshold model all of the peripheral area combines centrally. After combination, a central sensory threshold, $\Theta_{c}$, is assessed, such that only the above-central-threshold area determines performance.

Now consider a dichoptic presentation in which the $80-\mathrm{ms}$ duration is broken into two 40 -ms presentations, the first to one eye, and the second to the other eye with a zero ISI. ${ }^{6}$ The peripheral and central sensory threshold models yield different predictions for this condition. The middle panel of Figure 4 shows the central sensory response curve prediction for the peripheral threshold model. Only the above-peripheralthreshold area contributes to the central sensory response curve, but all of the central-sensory-response-curve area contributes to performance. Because the area reaching the central sensory response curve is smaller than the abovethreshold area in the monocular condition, the peripheral threshold model predicts dichoptic performance to be lower than monocular performance. This can be seen clearly in the middle panel of Figure 4 as a notch in the central sensory response curve of the dichoptic presentation. This loss of area results from two peripheral thresholds being assessed separately on two different peripheral sensory response curves, compared with the single peripheral threshold assessed on a single peripheral sensory response curve in the monocular case.

As we show next, a finding of poorer dichoptic performance than monocular performance not only is consistent with a peripheral threshold model but also disconfirms any model in which the sole threshold is central.

The right panel of Figure 4 shows the central sensory response curve for a dichoptic presentation predicted from the central sensory threshold model. The dashed curves are the peripheral sensory response curves before they combine to produce the central sensory response curve. Because the two peripheral sensory response curves combine before the central sensory threshold, the dichoptic central sensory response curve is identical to the monocular central sensory response curve, and they have the same above-centralthreshold area. Thus the central threshold model predicts monocular and dichoptic performance to be identical. It is for this reason that a finding of poorer dichoptic performance than monocular performance disconfirms a purely central threshold model.

\section{Predictions: Monocular Versus Binocular Conditions}

A second set of predictions involves comparison of the monocular and binocular conditions. In general, the means

\footnotetext{
${ }^{6}$ Because the stimuli were displayed on a computer monitor, the actual ISI was equal to the monitor's refresh rate, or $15 \mathrm{~ms}$. However, the monocular and dichoptic displays all had the same presentation time course (e.g., 6 refreshes to one eye, versus 3 refreshes to one eye followed by 3 refreshes to the other eye). Given this fact, we can safely assert that the effective ISI was zero, a presumption we will make throughout the rest of this article. Appendix C of Busey and Loftus (1994) discusses this issue in detail.
} 

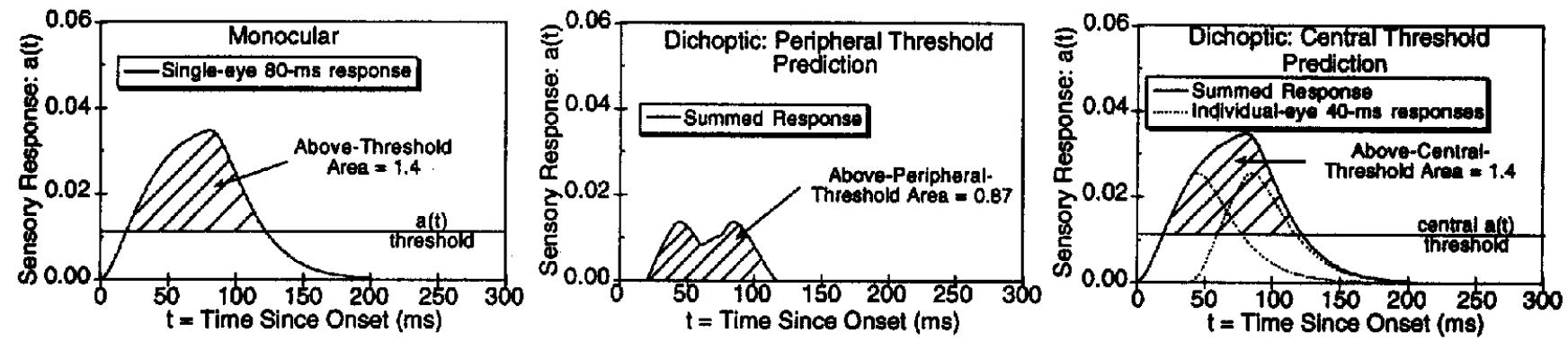

Figure 4. Left panel: Sensory response function for a monocular $d$-ms presentation. Middle panel: Peripheral threshold model's prediction for dichoptic presentation, which gives less area and thus reduced performance compared with the monocular condition. Right panel: Central threshold model's prediction for dichoptic presentation, which is identical to its prediction for monocular presentation, and thus dichoptic performance will be identical to monocular performance.

by which information from the two eyes is combined to produce binocular information-and, correspondingly, the relation between performance based on a monocular versus a binocular presentation-is the source of some debate (Blake \& Fox, 1973; Blake et al., 1981; Legge, 1984b). Two reasonable propositions have been put forth: first that the combination occurs via probability summation, and second that the combination occurs via quadratic summation. In our General Discussion we evaluate a third proposition that assumes neural interaction between the two ocular channels in a binocular presentation.

The supposition that the information combines by means of probability summation is easily incorporated in our theory. In what follows we develop a version of the theory that gives a prediction consistent with probability summation, and to do so we begin with probability summation and work backward. We are assuming that the two opportunities to view a binocular stimulus are equally affected by a sensory nonlinearity, and thus we are considering a version of the model that consists only of a peripheral threshold (although the same arguments below would hold assuming no thresholds).

Consider any two information sources (e.g., first and second presentations, left eye and right eye, etc.) and denote the proportions correct based on these two sources as $p_{1}$ and $p_{2}$. If $p_{\mathrm{B}}$ denotes proportion correct given both information sources, then probability summation implies that

$$
p_{\mathrm{B}}=p_{\mathrm{L}}+\left(1.0-p_{\mathrm{L}}\right) p_{\mathrm{R}}
$$

or

$$
\left(1.0-p_{\mathrm{B}}\right)=\left(1.0-p_{\mathrm{L}}\right)\left(1.0-p_{\mathrm{R}}\right) .
$$

Substituting $1.0-e^{-A \theta(\infty) / c}$ s (Equation 7) for each $p$ and simplifying, we find

$$
e^{-A_{\Theta, \mathrm{B}}(\infty) / c_{s}}=\left(e^{-A_{\theta, \mathrm{L}}(\infty) / c_{s}}\right)\left(e^{-A_{\theta, \mathrm{R}}(\infty) / c_{s}}\right)
$$

where $A_{\theta, \mathrm{L}}(\infty)$ and $A_{\Theta, \mathrm{R}}(\infty)$ represent the peripheral sensory response curves from the left and right eyes, respectively, after a peripheral threshold has been assessed. $A_{\Theta, B}(\infty)$ represents the sensory response function for both eyes. Taking the natural $\log$ of each side of the equation,

$$
A_{\Theta, \mathrm{B}}(\infty)=A_{\Theta, \mathrm{L}}(\infty)+A_{\Theta, \mathrm{R}}(\infty) .
$$

Thus Equation 10 may be interpreted to mean that two separate areas (e.g., two areas corresponding to performance from the two eyes separately) sum to produce a total area corresponding to performance in the combined (e.g., binocular) condition. Thus probability summation entails simply summing the two peripheral sensory response curves over $t$ to generate the central sensory response curve. Assuming equal contributions from both eyes, that is, $A_{\theta, \mathrm{L}}(\infty)=$ $A_{\Theta, \mathrm{R}}(\infty)=A_{\Theta, \mathrm{M}}(\infty)$, then $A_{\Theta, \mathrm{B}}(\infty)=2 A_{\Theta, \mathrm{M}}(\infty)$ and

$$
p_{\mathrm{B}}=2 p_{\mathrm{M}}-p_{\mathrm{M}}^{2}
$$

where $p_{\mathrm{B}}$ and $p_{\mathrm{M}}$ correspond to binocular and monocular performance, respectively. We stress that this assumes that the sensory threshold $\Theta$ is assessed before the site of combination and that the information-processing rates $c_{s}$ are the same for both eyes. Thus we are considering the peripheral threshold model for the moment.

We began this section by using the probability summation equation (Equation 9) and worked backward to produce a version of the theory that is consistent with Equation 9. The goal was to detail those assumptions necessary to produce the probability summation prediction. To summarize, if, in addition to the assumptions embodied in Equations 4, 5, and 6 , we also assume a linear combination mechanism and no postcombination information loss, then the theory will make a prediction that is consistent with probability summation (Equation 11). This is because the above-threshold area engendered by a binocular presentation is twice that engendered by a monocular presentation under a peripheral threshold model.

We have developed a model that makes a prediction consistent with probability summation but uses a linear summation combination rule. This may come as a surprise to some readers, who associate an or combination rule with the probability summation model (the stimulus is detected if the 
left eye detects it or if the right eye detects it, or both). A linear summation rule is typically associated with neural summation, which gives a prediction for binocular performance above that predicted by probability summation. How then can we develop a model with a linear summation rule that still predicts probability summation? The answer to this apparent paradox lies in the equation for converting abovethreshold area to performance (Equation 7). The exponential in this equation allows us to re-express the traditional probability summation equation (Equation 9) in terms of the sums of areas, which implies a linear summation mechanism. However, this formulation is formally equivalent to the or rule of probability summation. Probability summation contains the intrinsic notion of diminishing returns: If two observers or eyes each have a $50 \%$ chance of detecting the stimulus, the stimulus will be detected $75 \%$ of the time. Our formulation moves this notion of diminishing retums to the exponential in the information-processing stages. If we instead computed individual probabilities for the left and right eyes with Equation 7, we would have to adopt the or rule expressed in Equation 9 as our combination mechanism. Thus our adoption of a linear summation mechanism depends on the information-extraction portion of the theory (Equation 7). We should note that this component of the model is essential in that it accounts for performance as a function of exposure duration.

Note that the probability summation prediction is a consequence of the assumptions of (a) a linear summation mechanism, (b) no postcombinatorial information loss, and (c) our exponential information-extraction mechanism. Other models have adopted a linear summation mechanism and other assumptions and derive a different prediction of binocular performance on the basis of monocular data.

\section{Linear Summation Does Not Imply a Probability Summation Prediction}

At this point we wish to clearly distinguish between a probability summation prediction, which is a prediction of binocular performance based on monocular data, and a theory that assumes a linear-summation combination mechanism. The latter is a model of how the information from the two eyes combines. When a linear summation mechanism is conjoined with additional assumptions of no postcombinatorial information loss and an independent sampling information-acquisition mechanism, then a linear summation mechanism will make a prediction that is consistent with probability summation. However, as we show below, a theory that assumes a linear-summation combination mechanism will make predictions that differ from probability summation if a different set of assumptions about thresholds is made. Thus the question of combination mechanisms is intimately tied to the question of threshold locations; one cannot simply compare monocular and binocular data and infer the combinatorial mechanisms without also considering pre- and postcombination information losses. This is a major theme of the current work. We shall refer to a model that sums the information from the two eyes as a linearsummation combination mechanism, which under certain assumptions will predict probability summation but which under other assumptions will not.

When we refer to predictions of binocular data based on monocular performance, we will call this a probability summation prediction. When we refer to a specific model of binocular information combination that explicitly assumes a linear-summation combination mechanism and may, under certain assumptions, make a prediction of binocular performance that is consistent with probability summation, we shall refer to this as a linear summation model.

We are now in a position to discuss the model's predictions about the relation between the monocular and the binocular conditions. First consider the predictions for a peripheral threshold model, as shown in the top panel of Figure 5. In a monocular presentation, the digits appear in only one eye and generate only a single peripheral sensory response curve. The area that survives the peripheral threshold constitutes the central sensory response curve.

In a binocular presentation, the digits are presented to both eyes and generate two peripheral sensory response
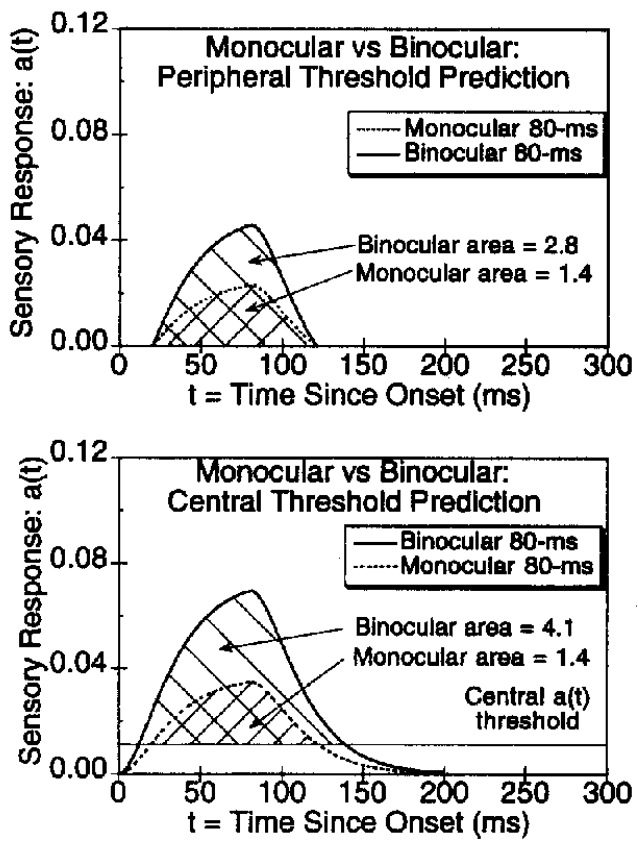

Figure 5. Top panel: Binocular and monocular central sensory response curves under a peripheral threshold model. The binocular area is always twice as great as the associated monocular area, and thus binocular and monocular performances obey a probability summation prediction: $p_{\mathrm{B}}=2 p_{\mathrm{M}}-p_{\mathrm{M}}^{2}$. Bottom panel: Binocular and monocular central sensory response curves under a central threshold model. Again the binocular curve is twice as tall as a monocular curve, but the central sensory threshold affects the monocular area proportionately more. This causes a failure of probability summation because binocular performance would be predicted to be greater than that expected from a probability summation prediction based on monocular performance: $p_{B}>2 p_{M}$ $-p_{\mathrm{M}}^{2}$. Under an extreme case, the monocular curve might not even reach the central sensory threshold, although a binocular curve might. In this case, $p_{\mathrm{M}}=0$ while $p_{\mathrm{B}}>0$, which is a clear failure of probability summation. 
curves. These two curves will both pass through peripheral sensory thresholds, and because the contrast of the two presentations is always the same, the two peripheral curves will be identical. ${ }^{7}$ As a result, the binocular condition's central sensory response curve will always be twice as high as the monocular condition's central sensory response curve, and thus the binocular area will always be twice as great. This implies that under a peripheral threshold model, binocular performance will fulfill a probability summation relation with monocular performance: $p_{\mathrm{B}}=2 p_{\mathrm{M}}-p_{\mathrm{M}}^{2}$. Note that this is in accordance with the probability summation prediction of Equation 11, although we have now described a specific model that makes a probability summation prediction. This model makes two key assumptions: no postcombinatorial source of information loss and a linear-summation combinatorial mechanism.

By the central threshold model, however, binocular performance is predicted to be greater than the binocular performance predicted by probability summation. The logic underlying this assertion is illustrated in the bottom panel of Figure 5. In the absence of peripheral thresholds, the two curves are the original monocular and binocular sensory response curves. As in the top panel, the binocular curve is overall twice as tall as the monocular curve. However, there is now a central sensory threshold, and only the above-centralthreshold area determines performance.

Note that much of the monocular area falls below the central threshold, leaving only an area of 1.4 above threshold in this example. In contrast, the binocular curve, by virtue of being higher than its monocular counterpart, is relatively less affected by the same threshold; hence the binocular above-threshold area (4.1 in this example) is greater than twice the monocular above-threshold area (of 1.4). This implies binocular performance above that predicted by probability summation (i.e., $p_{\mathrm{B}}>2 p_{\mathrm{M}}-p_{\mathrm{M}}^{2}$ ). This above-probability-summation prediction holds for any nonzero central sensory threshold (except for the trivial case when neither curve exceeds threshold and performance is zero in both conditions). This above-probability-summation prediction is a direct result of the central sensory threshold.

\section{Prediction Summary}

Let us summarize two critical predictions for Experiment 1. First, a finding that monocular performance exceeds dichoptic performance disconfirms a purely central threshold model and confirms a peripheral threshold model. Second, a finding that binocular performance exceeds what would be predicted on the basis of probability summation from the monocular condition disconfirms a purely peripheral model and confirms a central threshold model. Table 1 lists the assumptions and predictions for the peripheral and central threshold models.

\section{Method}

There were three main conditions in Experiment 1. In the first (monocular) condition a single $d$-ms stimulus was displayed to only one eye. In the second (dichoptic) condition there were two stimulus presentations, each $d / 2 \mathrm{~ms}$ in duration, separated by a
0 -ms ISI. The first $d / 2 \mathrm{~ms}$ of the stimulus was displayed to one eye, while the second $d / 2 \mathrm{~ms}$ of the stimulus was displayed to the opposite eye. In the third (binocular) condition a $d$-ms stimulus was presented to both eyes simultaneously.

\section{Observers}

Three experienced vision researchers participated in Experiment 1. Observer T.B. is Thomas A. Busey, and Observers G.W. and T.A.K. were visiting faculty members.

\section{Stimuli and Apparatus}

Stimulus presentation (and response collection) in Experiments 1, 2, and 3 were carried out on a Macintosh II computer. Stimuli were displayed on an Apple Monochrome monitor in conjunction with a Modified Wheatstone mirror stereoscope (see Blake \& Fox, 1973). The refresh rate $(67 \mathrm{~Hz})$ of the display device was sufficiently fast to eliminate flicker and poses little interpretational problem for our theory (see Busey \& Loftus, 1994, Appendix C).

Observers sat approximately $57 \mathrm{~cm}$ away from the screen in a dimly lit room and used the computer keypad to respond. A chin rest positioned the observer's head in front of two mirror arrangements that projected each half of the screen to one of the observer's eyes. An enclosing box eliminated reflections from the screen and assisted with eye fusion.

Considerable effort was made at the start of each block of trials to fuse the two halves of the monitor into one image without eye strain. Observers adjusted each of the four mirrors until the fixation point fused into a single image. They then adjusted the mirrors to create a cross out of two right angles, each of which was shown only to one eye. Figure 6 shows the experimental apparatus and the image used to adjust the mirrors. To further enhance eye fusion within a session, the background was a uniform gray field, and the fixation point never disappeared.

The digits were presented in the 24-point Times Roman font. The digits were each $0.50^{\circ}$ high by $0.40^{\circ}$ wide, separated by $0.75^{\circ}$ vertically and $0.40^{\circ}$ horizontally. Table 2 lists the luminances and contrasts. We used darker letters on a lighter background to avoid phosphor-decay problems. We programmed the experiment using a timing and display package described by Ames and Palmer (1992).

\section{Design and Procedure}

There were 8 monocular and dichoptic stimulus durations, ranging from 30 to $240 \mathrm{~ms}$ in 30 -ms increments. In the monocular condition, the entire $d$-ms duration was shown to either the right or left eye. In a corresponding dichoptic condition, $d / 2 \mathrm{~ms}$ of a $d$-ms

\footnotetext{
${ }^{7}$ The statement that both eyes contribute identical responses is a simplification, given that most observers have a dominant eye. However, any performance asymmetry between the two will simply bring binocular performance closer to the averaged monocular performance. Consider the extreme case in which an observer is blind in one eye. In this case, binocular performance will be exactly double the across-eye averaged monocular performance: Binocular performance will equal performance from the sighted eye, which is then averaged with zero performance from the blind eye. Our argument for the existence of a central threshold requires that binocular performance be greater than twice the monocular performance. However any asymmetry serves to reduce the difference between monocular and binocular performance and cannot increase the difference between binocular and averagedmonocular perfornance.
} 
Table 1

Assumptions and Predictions for the Four Models

\begin{tabular}{|c|c|c|}
\hline Model & Assumptions & Predictions \\
\hline $\begin{array}{l}\text { Peripheral threshold } \\
\text { model }\end{array}$ & $\begin{array}{l}\text { Each eye engenders a peripheral } \\
\text { response } a_{\mathrm{p}}(t) \text { which is sub- } \\
\text { jected to a peripheral } \\
\left.\text { threshold } \Theta_{\mathrm{p}} \text { (see Equation } 4\right) \\
\text { to create } a_{\theta, \mathrm{p}}(t) \text { for each eye. } \\
\text { These sensory response func- } \\
\text { tions are then summed to } \\
\text { create } a_{\mathrm{C}}(t) \text {. }\end{array}$ & $\begin{array}{l}\text { Dichoptic performance will be } \\
\text { below monocular perfor- } \\
\text { mance. } \\
\text { Binocular performance will } \\
\text { equal the probability summa- } \\
\text { tion prediction based on } \\
p_{\mathrm{B}}=2 p_{\mathrm{M}}-p_{\mathrm{M}}^{2} \text {. }\end{array}$ \\
\hline Central threshold model & $\begin{array}{l}\text { Each eye engenders a peripheral } \\
\text { response } a_{\mathrm{p}}(t) \text {, and these are } \\
\text { summed to create } a_{\mathrm{C}}(t) \text {. This } \\
\text { central sensory response func- } \\
\text { tion is then subjected to a cen- } \\
\text { tral sensory threshold } \Theta_{\mathrm{c}} \text { to } \\
\text { create } a_{\Theta, c}(t) \text {. }\end{array}$ & $\begin{array}{l}\text { Dichoptic performance will } \\
\text { equal monocular performance. } \\
\text { Binocular performance will be } \\
\text { greater than the probability } \\
\text { summation prediction based } \\
\text { on } p_{\mathrm{B}}=2 p_{\mathrm{M}}-p_{\mathrm{M}}^{2} \text {. }\end{array}$ \\
\hline Dual threshold model & $\begin{array}{l}\text { Each eye engenders a peripheral } \\
\text { response } a_{\mathrm{p}}(t) \text {, which is sub- } \\
\text { jected to a peripheral } \\
\left.\text { threshold } \Theta_{\mathrm{p}} \text { (see Equation } 4\right) \\
\text { to create } a_{\theta_{\mathrm{p}}}(t) \text { for each eye. } \\
\text { These are summed to create } \\
a_{\mathrm{c}}(t) \text {. This central sensory } \\
\text { response function is then sub- } \\
\text { jected to a central sensory } \\
\text { threshold } \Theta_{\mathrm{c}} \text { to create } a_{8, \mathrm{c}}(t) \text {. }\end{array}$ & $\begin{array}{l}\text { Dichoptic performance will be } \\
\text { below monocular perfor- } \\
\text { mance. } \\
\text { Binocular performance will be } \\
\text { greater than the probability } \\
\text { summation prediction based } \\
\text { on } p_{\mathrm{B}}=2 p_{\mathrm{M}}-p_{\mathrm{M}}^{2} \text {. }\end{array}$ \\
\hline Modified Cogan model & $\begin{array}{l}\text { Each eye engenders a peripheral } \\
\text { response } a_{\mathrm{p}}(t) \text {, which receives } \\
\text { inhibition from the contralat- } \\
\text { eral eye (see Equation } 15) \text {. } \\
\text { These are summed to create } \\
\text { the combined either-eye } \\
\text { output, } b_{x} \text {. A separate fused } \\
\text { channel } b_{\mathrm{F}} \text { creates the bin- } \\
\text { ocular component. The central } \\
\text { sensory response function } \\
a_{C}(t) \text { is the sum of } b_{x} \text { and } b_{\mathrm{F}} \text {. }\end{array}$ & $\begin{array}{l}\text { Dichoptic performance will be } \\
\text { below monocular perfor- } \\
\text { mance. } \\
\text { Binocular performance will be } \\
\text { greater than the probability } \\
\text { summation prediction based } \\
\text { on } p_{\mathrm{B}}=2 p_{\mathrm{M}}-p_{\mathrm{M}}^{2} \text {. However, } \\
\text { the magnitude of binocular } \\
\text { superiority over this predic- } \\
\text { tion may not be sufficient to } \\
\text { account for the data. }\end{array}$ \\
\hline
\end{tabular}

presentation was shown to either the right or left eye, followed immediately by the second (d/2)-ms presentation to the opposite eye, with a 0-ms ISI. Eye position was randomized across all conditions. In the binocular condition, there were 7 durations,

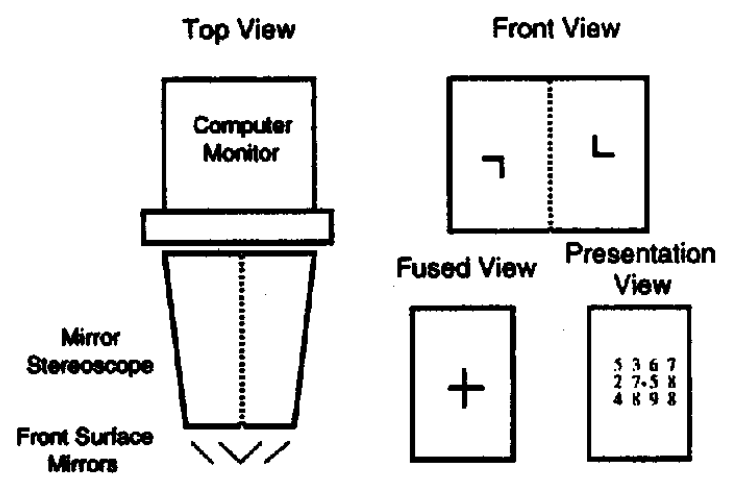

Figure 6. Display apparatus used in Experiments 1, 2, and 3. Care was taken at the start of a block of trials to fuse the two $L S$ into a cross to ensure that during the presentation view the digits overlapped on the same retinal area. The fixation point never disappeared, which allowed maintenance of the fused condition. ranging from $30 \mathrm{~ms}$ to $119 \mathrm{~ms}$ in $15-\mathrm{ms}$ increments. ${ }^{8}$ During monocular and dichoptic presentations, the eye opposite the stimulated eye saw the blank background field.

Observers completed eighteen 72-trial blocks, which provided 54 replications of each condition per observer."

\section{Results}

Figure 7 shows the data for Experiment 1, along with the predictions from the peripheral threshold model. Details of the parameter-fitting procedures and equations are provided

\footnotetext{
${ }^{8}$ We used different durations for monocular and binocular stimuli because we attempted to map out approximately the same performance ranges for both types of stimuli.

The experimental design for Experiment 1 originally called for 8 durations for each of the three conditions. However, we verified the stimulus presentation with a fast photometer and an oscilloscope, and we discovered that a software error had caused the 15-ms binocular condition to remain on the screen for $30 \mathrm{~ms}$. As a result, the data for this condition were averaged with the data for the $30-\mathrm{ms}$ condition, which gave 108 replications of the $30-\mathrm{ms}$ binocular condition.
} 
Table 2

Luminances and Contrasts for All Experiments

\begin{tabular}{lcc}
\hline $\begin{array}{l}\text { Luminances } \\
\text { and contrast }\end{array}$ & Digits & Fixation point \\
\hline & Experiments 1 and 2 \\
Background & 3.502 & 3.502 \\
Foreground & 3.252 & 1.472 \\
Contrast & 0.037 & 0.408 \\
\hline \multicolumn{3}{c}{} \\
Background & Experiment 3 \\
Foreground & 8.521 & 8.521 \\
Contrast & 7.392 & 2.011 \\
\hline
\end{tabular}

Note. All luminances are in candles $/ \mathrm{m}^{2}$. Contrast is defined as $\left(L_{\mathrm{t}}-L_{\mathrm{f}}\right) /\left(L_{\mathrm{b}}+L_{\mathrm{f}}\right)$, where $L_{\mathrm{b}}$ is the background luminance, and $L_{\mathrm{f}}$ is the foreground luminance.

in Appendix A. No notable performance differences exist for the 3 observers, and thus the data shown are averaged across observers. The upper (binocular) solid line represents the model's prediction of binocular performance based on probability summation from the monocular condition. Error bars represent standard errors, and the RMSE at the lower right is the root mean square error between the data points and the predicted values. ${ }^{10}$

Two findings and associated conclusions are apparent. First, the finding that dichoptic performance is below monocular performance allows us to reject a pure central threshold model. Second, the finding that binocular performance is above that predicted by probability summation allows us to reject a pure peripheral threshold model.

\section{Discussion}

The failure of both single-threshold models to account for performance in all three conditions leaves open the possibility that there are both a peripheral and a central threshold. Let us summarize the logic underlying this assertion.

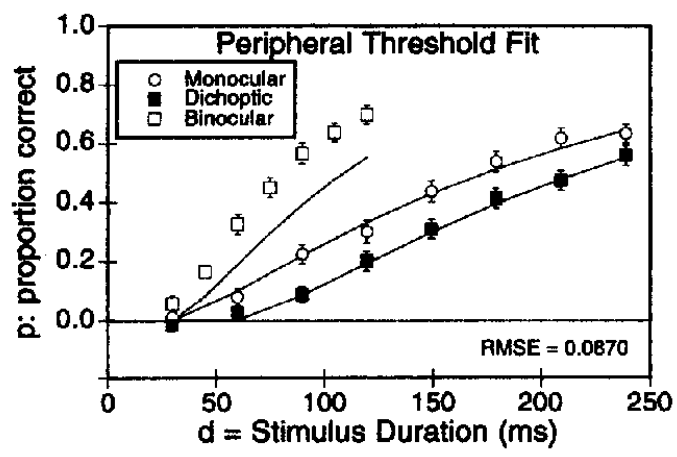

Figure 7. Experiment 1 results: Data are averaged for 3 observers along with the best-fitting predictions of the peripheral threshold model. For illustrative purposes, the model fit given in Figure 7 minimizes error around only the monocular and dichoptic presentations. This demonstrates the inadequacy of the probability summation assumption, implicit in the peripheral threshold model, for describing binocular performance based on monocular performance.
The observed binocular performance above that predicted from probability summation indicates that a complete model requires a central threshold. This central threshold hinders monocular performance more than binocular performance because the two monocular channels of a binocular presentation combine before the central threshold is assessed. The above-threshold binocular sensory response curve is therefore more than twice as high as an equal-duration monocular curve.

The observed inferiority of dichoptic performance relative to monocular performance indicates that a complete model must also include a peripheral threshold. The performance decrement for dichoptic presentations compared with monocular performance implies some form of information loss prior to the combination of the two ocular channels; such loss occurs with a peripheral threshold.

To summarize, we have rejected the pure forms of the peripheral and central sensory threshold models and concluded that both the peripheral and central sensory thresholds are necessary. We now alter our theory to account for these findings. Essentially, we do this by dividing the formerly single sensory threshold into peripheral and central sensory thresholds.

\section{The Dual Threshold Model}

Figure 8 illustrates this modified model, which we call the dual threshold model. Each ocular channel produces a sensory response function, complete with peripheral threshold. The above-threshold areas from each channel sum to produce the central sensory response function, which includes a central threshold.

$$
a_{\Theta, \mathrm{L}}(t)=\begin{array}{ll}
a(t)-\Theta_{\mathrm{p}} & \left(a(t)>\Theta_{\mathrm{p}}\right) \\
0 & \left(a(t) \leq \Theta_{\mathrm{p}}\right)
\end{array}
$$

where $a_{\theta, \mathrm{L}}(t)$ is the sensory response from the left eye after a peripheral threshold $\Theta_{p}$ is assessed. The response from the right eye, $a_{\Theta, \mathrm{R}}(t)$, is computed in the same fashion. These values combine to create a central sensory response function, $a_{c}(t)$,

$$
a_{\mathrm{c}}(t)=a_{\Theta, \mathrm{L}}(t)+a_{\Theta, \mathrm{R}}(t) .
$$

We then assess a central threshold,

$$
a_{\theta, \mathrm{c}}(t)=\begin{array}{ll}
a_{\mathrm{c}}(t)-\Theta_{\mathrm{c}} & \left(a_{\mathrm{c}}(t)>\Theta_{\mathrm{c}}\right) \\
0 & \left(a_{\mathrm{c}}(t) \leq \Theta_{\mathrm{c}}\right)
\end{array}
$$

where $a_{\theta, c}(t)$ represents the height of the sensory response

${ }^{10}$ The Figure 7 model fit differs from other model fits in that we minimized the model's predictions only on the monocular and dichoptic data, which demonstrates that although the peripheral threshold model can account for monocular and dichoptic performance, it cannot account for binocular performance. 

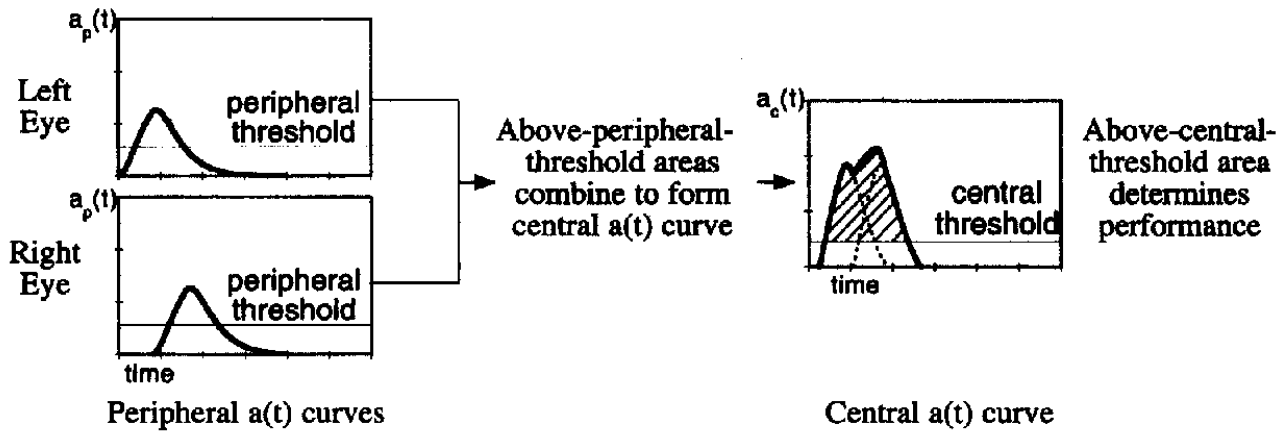

Figure 8. The dual threshold model. Above-peripheral-threshold areas from the peripheral sensory response curves combine to produce the central sensory response curve.

function after the central threshold has been assessed. $A_{\theta_{c}}(\infty)$ is the integral of $a_{\theta, \mathrm{c}}(t)$ from 0 to $\infty$, and performance is related to the above-central threshold area, $A_{\mathbf{\theta}_{c}}(\infty)$, by Equation 7.

Figure 9 shows the modified theory's best fit to the Experiment 1 data. The fit is extremely good, with an RMSE of 0.0189 . The assumption of a central threshold implies a model that contains a linear summation mechanism but no longer makes a probability summation prediction of $p_{\mathrm{B}}=$ $2 p_{\mathrm{M}}-p_{\mathrm{M}}^{2}$. Table 1 lists the assumptions and predictions for the dual threshold model.

Note that the site of information loss is relative to the combinatorial mechanisms. Information lost during the combination process, such as subadditive summation, would be considered postcombinatorial information loss because it would affect both the left- and right-eye information. Precombinatorial information losses affect only the information from a single eye.

Table 3 lists the parameter values for each observer and for the fit in Figure 9. The parameter values for the dual threshold model come from the temporal variables in Equation 1 and the threshold values from Equations 12 and 14. The information-processing rate parameter $c_{s}$ comes

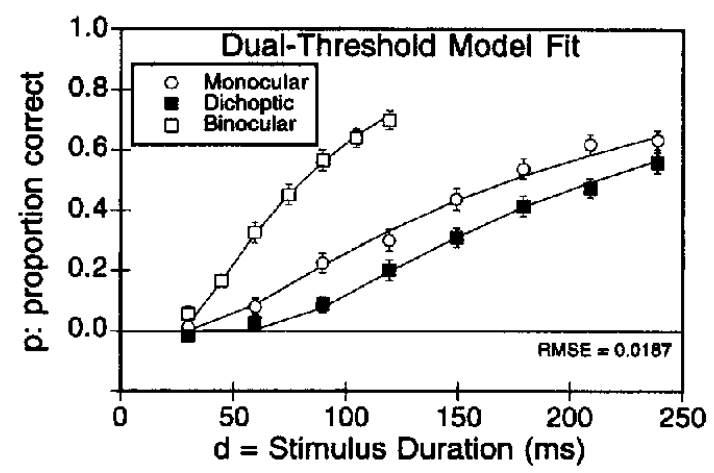

Figure 9. Experiment 1 results: Data are averaged for 3 observers; the solid line shows the best fit of the dual threshold model to the averaged data. An analysis of variance $F$ statistic comparing the mean squared deviation of the model $\left(\mathrm{RMSE}^{2}\right)$ to the average variability of each mean $\left(S E M^{2}\right)$ was not significant, $F(19,162)=$ $0.378, p>.05$, which supports the good fit of the model to the data. from Equation 7. We also provide the parameter values for the power-function version of the model, with the analogous peripheral and central $\beta$ values.

\section{Accounting for Previous Data}

Given the necessity of two thresholds to adequately describe the Experiment 1 data, we might ask how previous experiments using similar stimuli, such as those described in Loftus et al. (1993), Loftus and Ruthruff (1994), and Busey and Loftus (1994), could be fit by a single-threshold model. The answer is that, mathematically, the previously assumed single threshold subsumes both thresholds. The two thresholds simply combined and were represented by a single threshold. Only when the binocular and dichoptic conditions were included did the two thresholds reveal themselves. The dual threshold model would, of course, also fit the data from Loftus et al. (1993), Loftus and Ruthruff (1994), and Busey and Loftus (1994), albeit with a $100 \%$ trade-off between the two threshold parameters.

\section{Dual Threshold Model Predictions for Dichoptic and Gap-Monocular Presentations}

A further test of the dual threshold model can be made as follows. Experiment 4 of Busey and Loftus (1994) included the present monocular condition, as well as a related condition called a gap-monocular condition, in which the total duration $(d)$ of the monocular presentation was split into two temporal (d/2)-ms halves, separated by a $250-\mathrm{ms}$ blank screen. Observers performed substantially better in the monocular condition than in the gap-monocular condition. As we showed (Busey \& Loftus, 1994), this is predicted by the theory, which implies more sensory response area above threshold in the monocular than in the gap-monocular condition.

The dichoptic condition of Experiment 1 is analogous to a gap-monocular condition except for the 0-ms ISI and the presentation of each pulse to a different eye. Dichoptic condition performance is, like gap-monocular condition performance, worse than monocular performance. This is implied by the theory in part because a dichoptic condition suffers the effects of two peripheral thresholds, whereas a 
Table 3

Summary of Best-Fitting Dual Threshold Model and Dual Power-Function Model Parameters for Experiments 1-3

\begin{tabular}{|c|c|c|c|c|c|c|c|c|c|c|}
\hline \multirow[b]{2}{*}{ Observer } & \multicolumn{5}{|c|}{ Dual threshold model } & \multicolumn{5}{|c|}{ Dual power-function model } \\
\hline & $\tau$ & $c_{s}$ & $\boldsymbol{\Theta}_{\mathrm{P}}$ & $\overline{\Theta_{C}}$ & RMSE & $\tau$ & $c_{s}$ & $\mathbf{\theta}_{\mathbf{p}}$ & $\Theta_{c}$ & RMSE \\
\hline \multicolumn{11}{|c|}{ Experiment 1} \\
\hline $\begin{array}{l}\text { G.W. } \\
\text { T.B. } \\
\text { T.A.K. } \\
\text { Average }\end{array}$ & $\begin{array}{r}12.95 \\
7.27 \\
6.73 \\
8.02\end{array}$ & $\begin{array}{l}4.07 \\
2.37 \\
2.23 \\
3.11\end{array}$ & $\begin{array}{l}0.46 \\
1.26 \\
1.32 \\
0.97\end{array}$ & $\begin{array}{l}1.61 \\
1.18 \\
0.62 \\
1.10\end{array}$ & $\begin{array}{l}.0333 \\
.0329 \\
.0367 \\
.0189\end{array}$ & $\begin{array}{r}13.46 \\
7.27 \\
5.06 \\
6.13\end{array}$ & $\begin{array}{l}8.69 \times 10^{-3} \\
1.16 \times 10^{-4} \\
1.00 \times 10^{-4}= \\
1.65 \times 10^{-4}\end{array}$ & $\begin{array}{l}1.36 \\
2.26 \\
3.16 \\
2.54\end{array}$ & $\begin{array}{l}2.29 \\
1.92 \\
1.35 \\
1.70\end{array}$ & $\begin{array}{l}.0313 \\
.0382 \\
.0431 \\
.0206\end{array}$ \\
\hline \multicolumn{11}{|c|}{ Experiment 2} \\
\hline $\begin{array}{l}\text { G.W. } \\
\text { T.B. } \\
\text { T.K. } \\
\text { G.W.-No } \Theta_{C}{ }^{b} \\
\text { G.W.-No } \beta_{C}\end{array}$ & $\begin{array}{l}28.55 \\
24.56 \\
22.39 \\
22.16\end{array}$ & $\begin{array}{l}6.50 \\
5.15 \\
3.61 \\
6.10\end{array}$ & $\begin{array}{l}0.65 \\
0.21 \\
0.53 \\
1.02\end{array}$ & $\begin{array}{l}0.16 \\
0.55 \\
0.37\end{array}$ & $\begin{array}{l}.0324 \\
.0401 \\
.0498 \\
.0318\end{array}$ & $\begin{array}{r}8.39 \\
13.12 \\
7.99 \\
\\
8.74\end{array}$ & $\begin{array}{r}1.50 \times 10^{-6} \\
1.45 \times 10^{-2} \\
4.65 \times 10^{-6} \\
12.92 \times 10^{-5}\end{array}$ & $\begin{array}{l}1.98 \\
1.20 \\
1.48 \\
\\
5.06\end{array}$ & $\begin{array}{l}2.88 \\
2.35 \\
3.49\end{array}$ & $\begin{array}{l}.0288 \\
.0385 \\
.0485 \\
.0287\end{array}$ \\
\hline \multicolumn{11}{|c|}{ Experiment 3} \\
\hline $\begin{array}{l}\text { T.B. } \\
\text { L.M. } \\
\text { M.B. } \\
\text { Average }\end{array}$ & $\begin{array}{l}17.09 \\
15.78 \\
14.48 \\
15.33\end{array}$ & $\begin{array}{l}3.87 \\
2.25 \\
7.57 \\
4.38\end{array}$ & $\begin{array}{l}0.14 \\
0.68 \\
0.31 \\
0.32\end{array}$ & $\begin{array}{l}1.89 \\
0.85 \\
1.66 \\
1.41\end{array}$ & $\begin{array}{l}.0482 \\
.0362 \\
.0376 \\
.0262\end{array}$ & $\begin{array}{r}19.42 \\
2.98 \\
10.99 \\
12.22\end{array}$ & $\begin{array}{l}2.85 \times 10^{-2} \\
1.91 \times 10^{-6} \\
9.15 \times 10^{-2} \\
8.68 \times 10^{-2}\end{array}$ & $\begin{array}{r}1.01 \\
266.21 \\
1.17 \\
1.14\end{array}$ & $\begin{array}{l}2.74 \\
0.02 \\
2.32 \\
2.19\end{array}$ & $\begin{array}{l}.0512 \\
.0536 \\
.0324 \\
.0387\end{array}$ \\
\hline
\end{tabular}

Note. Threshold parameters $\Theta_{P}$ and $\Theta_{C}$ are in units of percentage contrast, $\theta$ and $c_{s}$ are in milliseconds, and the units for RMSE (root mean square error) are percentages of correctly recalled digits. Note that the RMSEs for combined data may not agree with the RMSEs in Figures $7,9,11,14$, and 16. Table 3 combined RMSEs are derived from parameter searches on averaged data, whereas the RMSEs in Figures 7, 9, 11,14 , and 16 are computed by averaging the individual model fits from the 3 observers and comparing these with the averaged data from the 3 observers. Thus the graphs in the figures represent averages of model fits fit to individual data rather than a fit to averaged data. $n=9$ for all fits.

T.A.K.'s Experiment 1 power-function model fit was best fit by a very small $c_{s}$ value coupled with a large $\beta_{\mathrm{P}}$ value. However, when $c_{s}$ was fixed to a reasonable value $(0.0001)$, the fit was not markedly worse. The best-fitting parameter values were $\tau=2.84, c_{s}=1.38 \times 10^{-21}$, $\beta_{P}=12.023, \beta_{C}=1.33$, and RMSE $=.0408$. bThe fit from Experiment 2 labeled G.W.-No $\theta_{C}$ represents the fit of the peripheral threshold model to Observer G.W. Because of differences among observers, we did not fit a version of the theory to averaged data.

monocular presentation suffers from only a single peripheral threshold.

The dichoptic and gap-monocular conditions are related in that in both, the stimulus is divided into two temporal halves, and performance in both conditions is worse than monocular performance. However, the dual threshold model predicts a performance difference between these two conditions. The reason is described in detail later, but briefly it is as follows. The dichoptic condition's 0-ms ISI allows the two peripheral sensory response curves to overlap and sum, and thus provides more above-central-threshold area under the central sensory response curve compared with a gapmonocular condition. The gap-monocular condition's large ISI prevents the two peripheral sensory response curves from overlapping, leaving less above-central-threshold area. Thus, on the basis of the slightly more area above the dichoptic curve's central sensory threshold, the dual threshold version of the theory predicts a slight performance advantage for the dichoptic condition compared with the gap-monocular condition. Expressed differently, it appears that we cannot treat two separate presentations to separate eyes as simply independent, to-be-summed events, as would be dictated by a simple, peripheral-threshold-only model. The dichoptic peripheral sensory response curves overlap and push more area above the central sensory threshold, whereas the two curves from the two pulses in a gapmonocular presentation cannot overlap. The extra area pushed above the central threshold predicts that dichoptic performance will be slightly above gap-monocular performance.

A comparison of our previous Experiment 4 (Busey \& Loftus, 1994) and the present Experiment 1 confirms this prediction: The difference between the dichoptic and monocular curves in the present Experiment 1 is not as great as the difference between the gap-monocular and monocular curves in our previous Experiment 4 (Busey \& Loftus, 1994). Subtracting gap-monoptic performance from monoptic performance reveals that $20-25$ percentage points separate the two conditions in our previous Experiment 4 (Busey \& Loftus, 1994). ${ }^{11}$ However, subtracting dichoptic performance from monocular performance reveals a separation of only 10-15 percentage points between the two conditions of Experiment 1. Monoptic performance was similar in the two experiments, never differing by more than $10 \%$ across experiments.

Although these findings suggest that gap-monoptic performance is inferior to dichoptic performance, this conclusion requires an across-experiment comparison. In Experiment 2 we replicated this finding by comparing the three conditions

11 The Busey and Loftus (1994) data were graphed using a nonlinear transformation of percentage correct, but here we converted the previous Experiment 4 data back to percentage correct to make this comparison. 
(monocular, dichoptic, and gap-monocular) in a single experiment. Thus Experiment 2 provides a further test of the dual threshold model along with independent estimates of the model parameters.

\section{Experiment 2: Testing the Independent Sampling Model With Two Thresholds}

From the perspective of the dual threshold model, the fundamental difference between a dichoptic and a gapmonocular presentation is that the two peripheral sensory response curves of a dichoptic presentation are processed independently only up to the point where they combine to produce the central sensory response function. After this happens, the two peripheral sensory response curves overlap and sum to produce more area above the central sensory threshold in a dichoptic presentation, and thus the model predicts greater dichoptic performance compared with a corresponding gap-monocular condition.

To see why this is so, consider Figure 10. The top panel shows the central sensory response curve for a $150-\mathrm{ms}$ monocular presentation with the central sensory threshold. The middle panel shows the central sensory response curve for a dichoptic presentation consisting of two 75-ms displays with a 0-ms ISI. Even though the overall exposure duration is identical in the two conditions, the top of the dichoptic sensory response curve appears bowed when compared with the monocular curve. This happens because presenting all of the stimulus to the same eye, as in a monocular presentation, creates a sensory response curve that is wider above the sensory threshold than are the two dichoptic sensory response curves (see left panel of Figure 10, solid vs. dashed curves). This results in more above-peripheral-threshold area for the monocular condition. The bow in the dichoptic central sensory response function (see middle panel of Figure 10) results from the narrower dichoptic peripheral sensory response curves, which tend to lose more area to the peripheral sensory threshold.

The bottom panel of Figure 10 shows the central sensory response curve for a gap-monocular presentation consisting of two 75 -ms displays separated by a 250-ms ISI. This panel demonstrates the sensory independence between the two curves: The ISI is long enough to prevent the first presentation from summing with the second. Accordingly, there is less area above the central sensory threshold, which reduces performance for this condition compared with both the monocular and dichoptic conditions.

The two peripheral sensory thresholds ensure that dichoptic performance is below monocular performance, but the interaction of the two dichoptic peripheral sensory response curves at the central sensory response curve drives more area above the central sensory threshold. Thus dichoptic performance is predicted to be above performance in the gap-monocular condition but below that in the monocular condition.

\section{Predictions Summary}

Let us summarize the predictions of Experiment 2. A finding of monocular performance above dichoptic perfor-
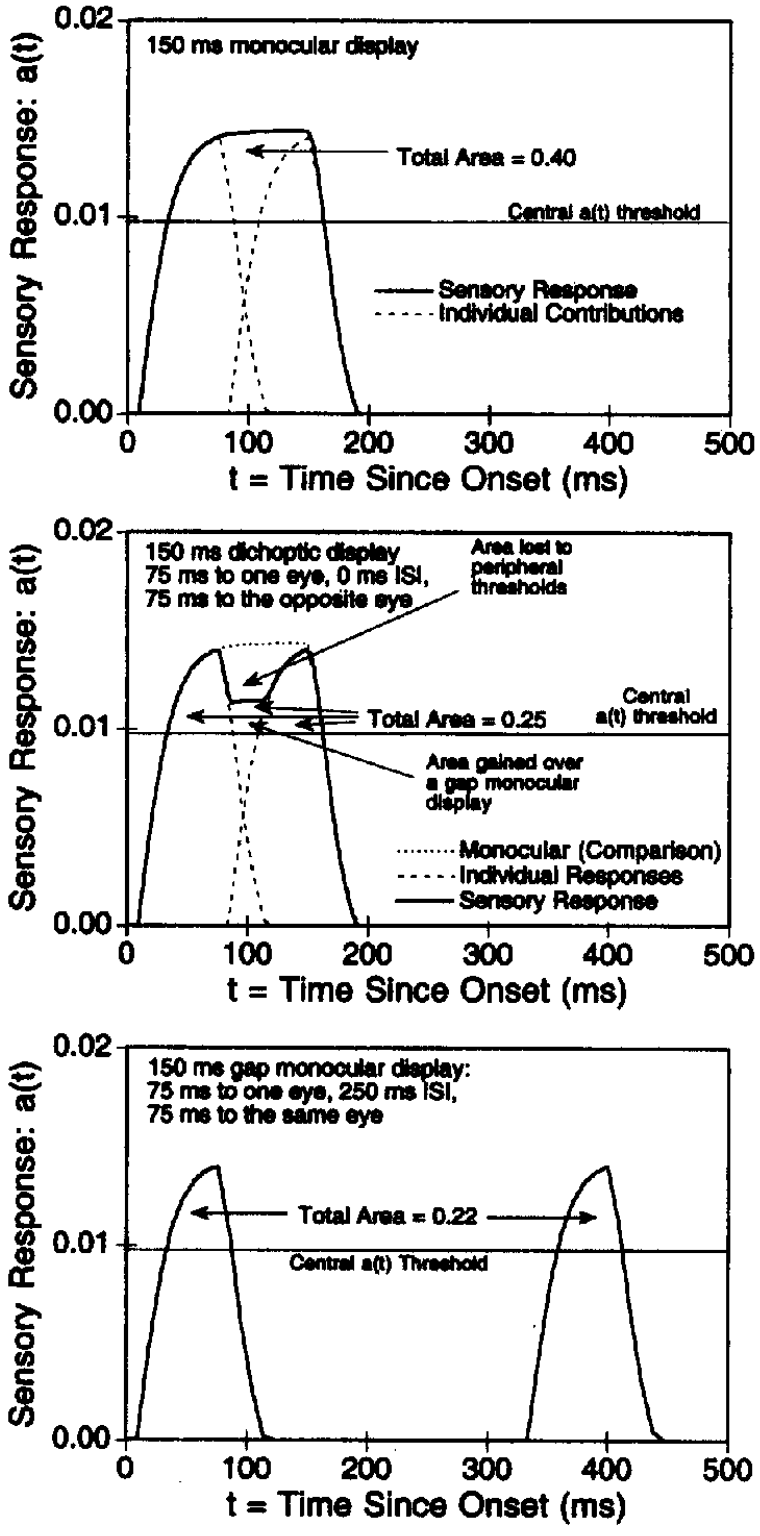

Figure 10. Top panel: Central sensory response curve for a 150-ms monocular presentation. For comparison with the dichoptic and gap monocular stimuli, an 80-ms monocular stimulus can be thought of as two $40-\mathrm{ms}$ presentations to the same eye with a 0-ms ISI. Middle panel: Central sensory response curve for a $150-\mathrm{ms}$ dichoptic presentation. Bottom panel: Central sensory response curve for a 150-ms gap-monocular presentation. The dichoptic and gap-monocular curves have less above-central-sensory-responsethreshold area than does the monocular curve, which predicts lower performance for the dichoptic and gap-monocular conditions. However, the dichoptic area is slightly greater than the gapmonocular area, which implies that dichoptic performance will be slightly above the gap-monocular performance. The monocular stimulus has an advantage over the dichoptic stimulus in that its two "halves" combine before a peripheral threshold is assessed, which pushes more area above the peripheral threshold. 
mance implies a model with a peripheral threshold. A finding of dichoptic performance above gap-monocular performance implies a central threshold.

\section{Method}

Experiment 2 incorporates three basic conditions. In the monocular condition a single $d$-ms stimulus was presented to one eye. In the dichoptic condition there were two (d/2)-ms stimulus presentations, the first to one eye and the second to the other eye, separated by a 0 -ms ISI. In the gap-monocular condition there were two (d/2)-ms presentations, both to the same eye, separated by a $250-\mathrm{ms}$ ISI.

\section{Observers, Stimuli, and Apparatus}

Observers, stimuli, and apparatus were identical to those of Experiment 1 except that Observer T.A.K. was replaced by Observer T.K., a male graduate student.

\section{Design and Procedure}

The design and procedure were essentially identical to those of Experiment 1 except that the binocular condition was replaced by the gap-monocular condition. There were eight durations with each of the three conditions. Observers completed eighteen 72-trial blocks, which provided 54 observations per condition per observer.

\section{Results}

Figure 11 shows the results of Experiment 2. Because there were qualitative differences between G.W. and the other 2 observers, we present individual data here. T.B.'s and T.K.'s data (left and middle panels) confirm the foregoing prediction: Dichoptic performance (filled squares) lies between monocular and gap-monocular performance. T.K.'s dichoptic data are close to the gap-monocular data, although dichoptic performance is significantly greater than the gap-monocular data, $t(1,520)=3.87, p<.0001$.

Observer G.W.'s data are shown in the right panel. The most important difference is that dichoptic and gapmonocular performance are the same. The dual threshold model, however, can fit each data pattern with different parameter values. Consider the comparison between $\mathrm{Ob}$ server T.B. and Observer G.W. Figure 12 shows the central sensory response curves for Observers T.B. and G.W. for each condition, as generated by the best-fitting model parameters. The top three panels demonstrate why a dual threshold model predicts dichoptic performance between the monocular and gap-monocular performances: The low peripheral threshold and the temporal proximity of the two peripheral sensory response curves combine to produce more above-threshold area relative to the gap-monocular condition.

Observer G.W.'s overall performance is approximately half that of the other 2 observers. From the model's perspective, this is a direct result of a high peripheral sensory threshold, which causes a greater loss of area compared with the performance of Observer T.B. The bottom three panels of Figure 12 show the central sensory response curves given by the best-fitting model para-
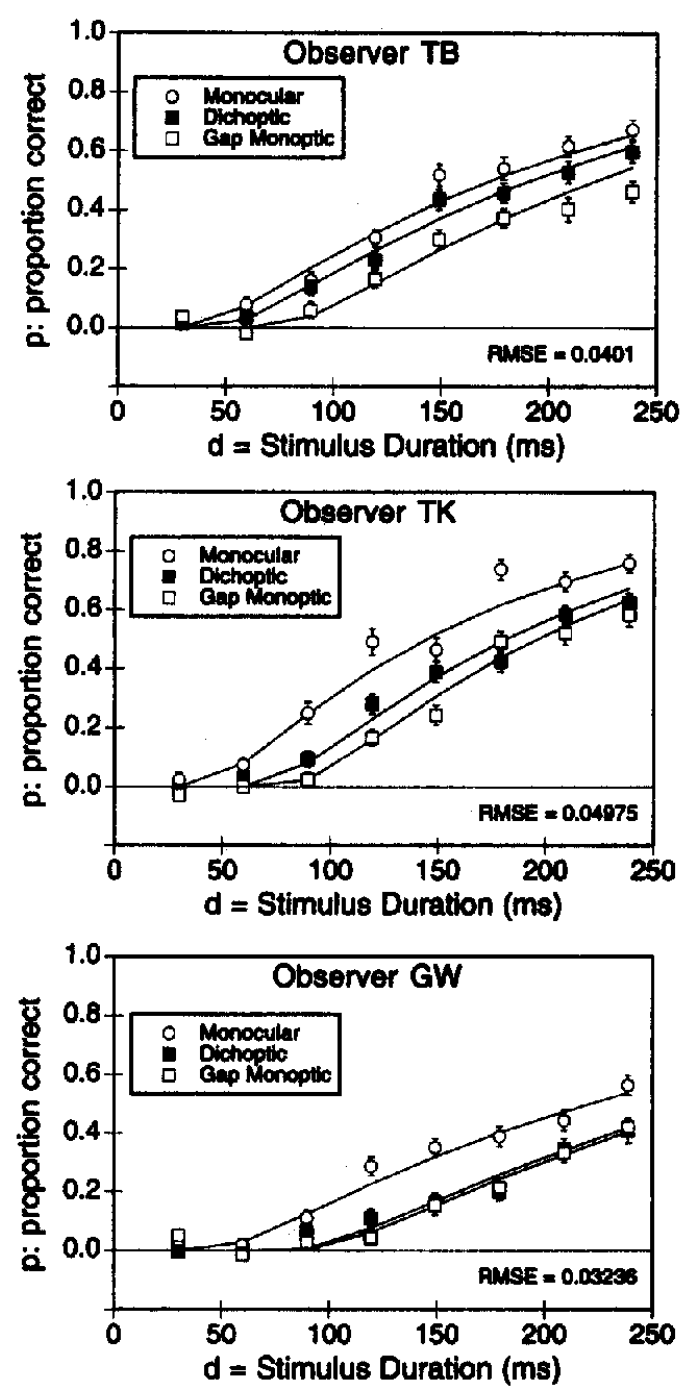

Figure 11. Experiment 2 results: data for 3 separate observers along with best fits from the dual threshold model. $F$ statistics computed as for the data in Figure 9 show that the model did not significantly deviate from the data for Observers T.B. and G.W., $F(20,54)=1.79, p>.05$ and $F(20,54)=1.32, p>.05$, whereas for Observer T.K. it did deviate significantly from the data, $F(20$, $54)=2.64, p<.05$, perhaps in part because of the variability in the monocular data.

meters for G.W. A large peripheral threshold does not allow the two peripheral sensory response curves to overlap centrally as much. Without this large overlap, the two dichoptic curves will produce about the same abovethreshold area as the gap-monocular curve and subsequently similar performance.

Because Observer G.W.'s dichoptic and gap-monocular performances were close together, a dual threshold model with the central threshold set to zero (equivalent to the pure peripheral threshold model) will account for the data. However, the best fit to the data comes from peripheral and central sensory thresholds that are both positive. In addition, 

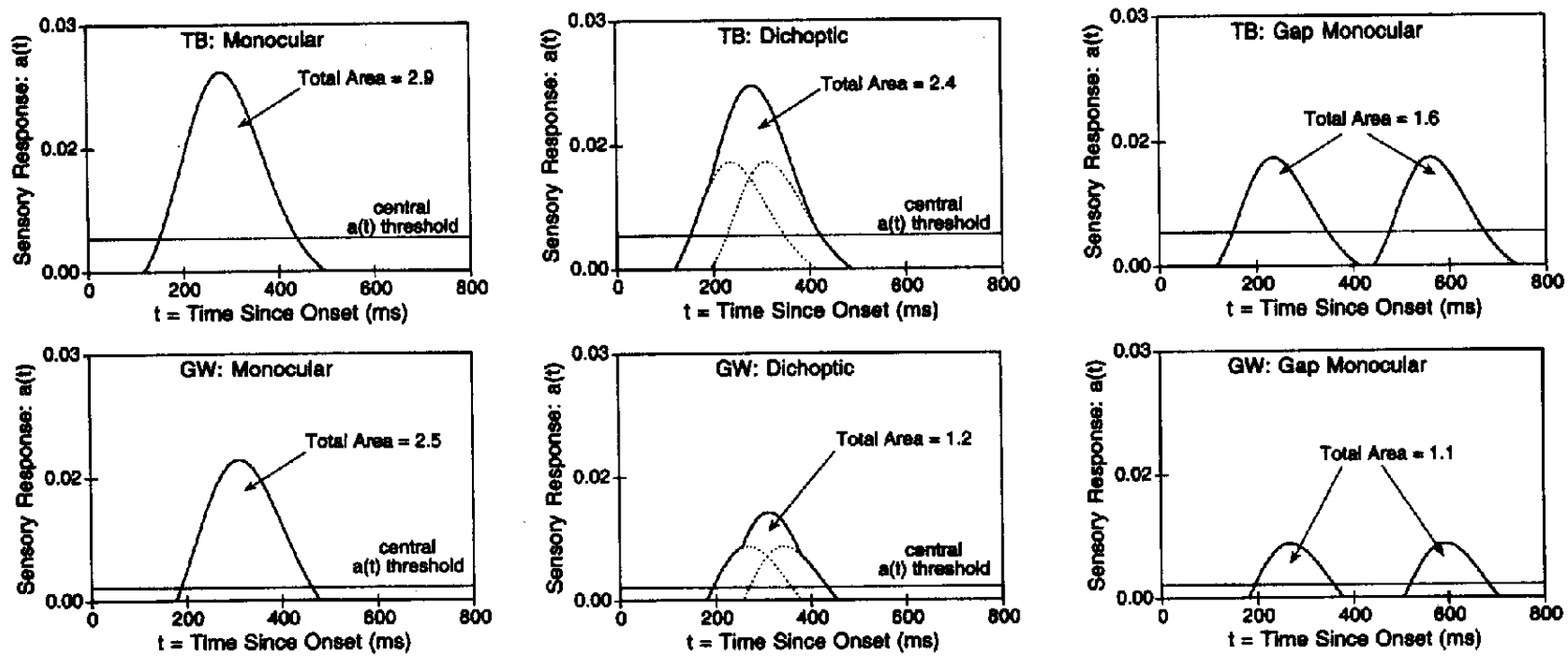

Figure 12. Central sensory response curves for Experiment 2 Observers T.B. and G.W., which show how different model parameters generate qualitatively different predictions with the same theory. G.W.'s large peripheral thresholds allow less overlap of the peripheral dichoptic curves (middle panel), and thus they do not provide much more above-central-threshold area than the above-central-threshold area derived from the gap-monocular condition.

Observer G.W.'s Experiment 1 data also required a central threshold to provide a good model fit.

Observer T.K.'s overall performance lies somewhere in between that of the other observers, and he has a peripheral threshold that is in between T.B.'s and G.W.'s. We see a concomitant reduction in the difference between dichoptic and gap-monocular performance. However, this data pattern is readily accounted for by the theory. The parameter values for the 3 observers in Experiment 2 are listed in Table 3.

\section{Discussion}

Experiment 2 confirms the dual threshold model, which is implied by T.B.'s and T.K.'s data, and provides the best fit to G.W.'s data. In addition, the fit to G.W.'s data demonstrates how predictions that seem qualitatively different come from a single theory's different parameter values.

\section{Experiment 3: Testing the Central Threshold}

Experiments 1 and 2 provided strong support for a peripheral threshold: Monocular performance above dichoptic performance requires some form of precombination information loss. This information loss is represented in the dual threshold theory by a peripheral threshold. All 3 observers in Experiments 1 and 2 demonstrated a clear superiority of monocular performance over dichoptic performance. However, Experiment 2 provides only weak support for a central threshold; only 2 of the 3 observers (T.B. and T.K.) demonstrated a difference between dichoptic and gap-monocular performance. Perhaps the conditions used in Experiment 2 were not powerful enough to distinguish between a theory that requires a central threshold and a theory that does not. One goal of ours in Experiment 3 was to test the central threshold component of the theory directly.

To test the central threshold component of the dual threshold theory, in Experiment 3 we incorporated dichoptically presented stimuli and variation in the ISI between the two pulses of a dichoptic presentation. To see how this manipulation identifies the existence of a central sensory threshold, consider a basic dichoptic presentation consisting of a $60-\mathrm{ms}$ presentation to one eye followed by a $60-\mathrm{ms}$ presentation of the same stimulus to the other eye. Consider two conditions that differ in the ISI between the two presentations. In the first condition, the ISI is $0 \mathrm{~ms}$, and in the second condition it is $45 \mathrm{~ms}$. Because the two conditions engender two identical peripheral sensory response curves, they both generate the same above-peripheral-threshold area passed to the combination mechanisms. As a result of these identical areas passed to the combination mechanism, any performance difference between the two conditions must be caused by postcombinatorial mechanisms; that is, a detrimental ISI effect must result from either a loss due to a central threshold or a loss in the combination of the two peripheral areas via nonlinear summation.

Figure 13 shows the central sensory response curves resulting from monocular, 0-ms-ISI dichoptic presentations and 45-ms-ISI dichoptic presentations. It demonstrates that a central threshold assumption makes the prediction that as ISI increases, performance will decrease in the dichoptic condition.

\section{Method}

In Experiment 3 we used the monocular and dichoptic stimuli of Experiments 1 and 2 . However, in the dichoptic condition, the ISI 

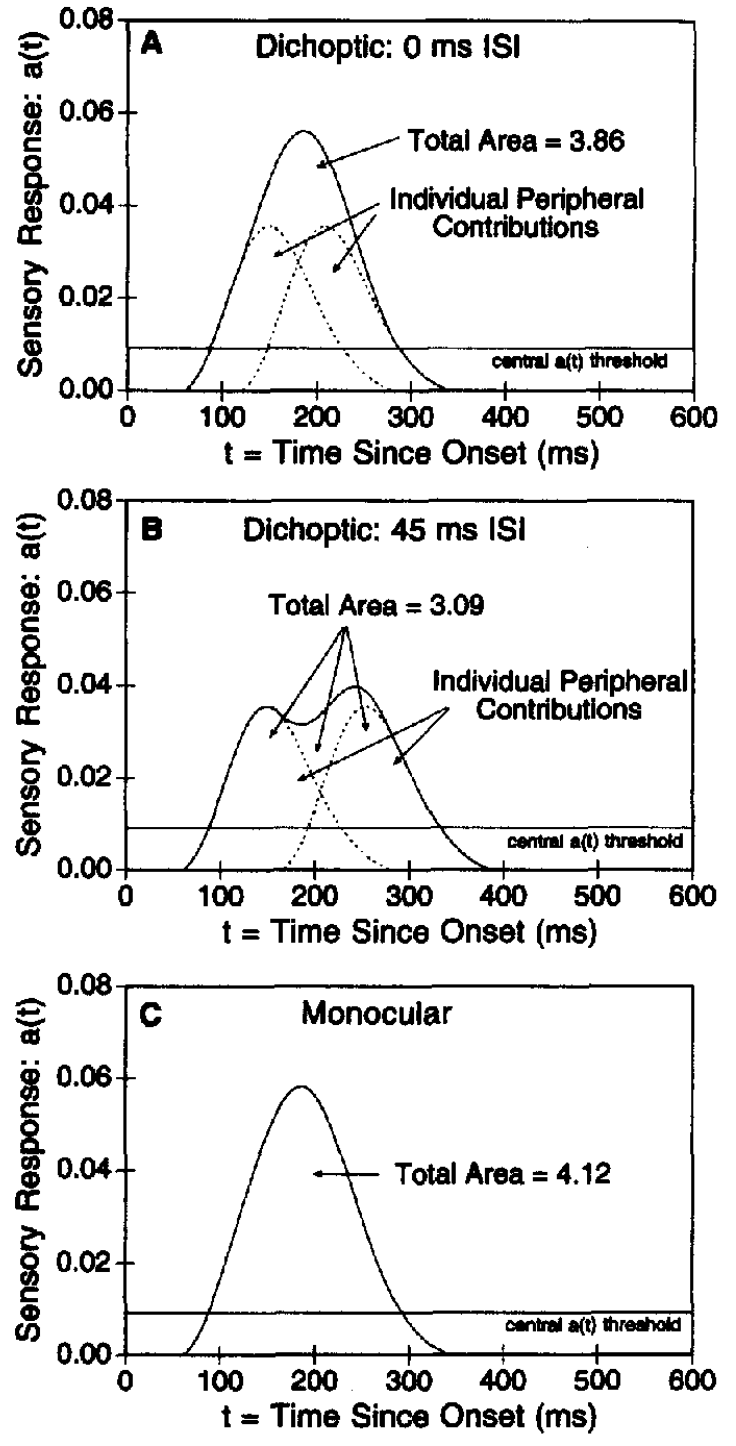

Figure 13. Linear-summation dual threshold model. Central sensory response curves for 0-ms-ISI dichoptic, 45-ms-ISI dichoptic, and monocular presentations for a $60-\mathrm{ms} / 60-\mathrm{ms}$ condition. The two dichoptic-condition central sensory response curves have the same overall area (Panels $A$ and $B$ ), because the individual peripheral contributions are the same for both conditions. However, because the $45-\mathrm{ms}-\mathrm{ISI}$ dichoptic condition has area that is more spread out in time, the central threshold affects it more, which gives it less above-central-threshold area (3.86 vs. 2.09 ). This leads to the prediction that as ISI is increased in the dichoptic condition, performance will decrease. This decrease is due solely to the central sensory threshold because the peripheral sensory thresholds affect both pulses equally in all conditions. Panel $\mathrm{C}$ shows the curve resulting from a monocular presentation; the monocular area is slightly larger than the 0-ms-ISI dichoptic area because the taller peripheral sensory response curve for the monocular condition puts more area above the peripheral sensory threshold. The combination of peripheral and central thresholds leads to the prediction that monocular performance will be above the 0-ms-ISI dichoptic performance (as was seen in Experiments 1 and 2) and that performance will decrease as ISI is increased in the dichoptic condition. between the first and second pulses was systematically varied from 0 to $90 \mathrm{~ms}$. The duration of each of two pulses in each dichoptic presentation was either $30,45,60$, or $75 \mathrm{~ms}$.

\section{Observers}

Three observers participated in Experiment 3: Thomas A. Busey (T.B.), a female graduate student (L.M.), and a male graduate student (M.B.).

\section{Stimuli and Apparatus}

The stimuli and apparatus were identical to those of Experiment 1. The contrast of the digits was set to $7.1 \%$.

\section{Design and Procedure}

Both monocular and dichoptic stimuli were used. Within the dichoptic condition were 5 different ISIs ranging from 0 to $90 \mathrm{~ms}$. The two pulses were the same duration, which was $30,45,60$, or 75 $\mathrm{ms}$. Within the monocular condition were four stimulus durations: $60,90,120$, or $150 \mathrm{~ms}$. Observers completed twenty-four 72-trial blocks, which provided 72 observations per condition per observer.

\section{Results and Discussion}

Experiment 3 results are summarized in Figure 14. All 3 observers produced data that demonstrate the same overall pattern, and thus averaged data are shown. The solid lines represent the fit of the dual threshold model, the best-fitting parameters of which are provided in Table 3 . Two findings in these data are noteworthy.

First, as ISI is increased in the dichoptic condition, performance decreases. This implies some form of postcombinatorial information loss, as modeled by a central sensory threshold in Figure 13. Prior to the combination of the information from each eye, dichoptic presentations with different ISIs all provide the same information; the ISI only becomes relevant once the responses from each eye are combined. However, under a linear summation mechanism, increasing the ISI still cannot cause a decrease in performance without something like a central sensory threshold, because the various dichoptic conditions have the same overall area under their central sensory response functions. The central threshold causes a reduction in performance for longer-ISI dichoptic conditions because it tends to eliminate more of the area under the wider, shorter central sensory response curves produced by a longer-ISI dichoptic condition. Thus under the current dual threshold model, the Figure 14 data imply a central sensory threshold.

The second noteworthy finding in the Figure 14 data is that monocular performance is above performance in the 0 -ms-ISI dichoptic condition. This is most clearly evident for the short-duration presentations. Overall, the magnitude of the effect is somewhat reduced when compared with the differences observed between these two conditions in Experiments 1 and 2. However, this pattern held for all 3 observers, with the exception of the longest-duration condition for Observer T.B. The superiority of monocular performance in this experiment as well as in Experiments 1 and 2 constitutes 


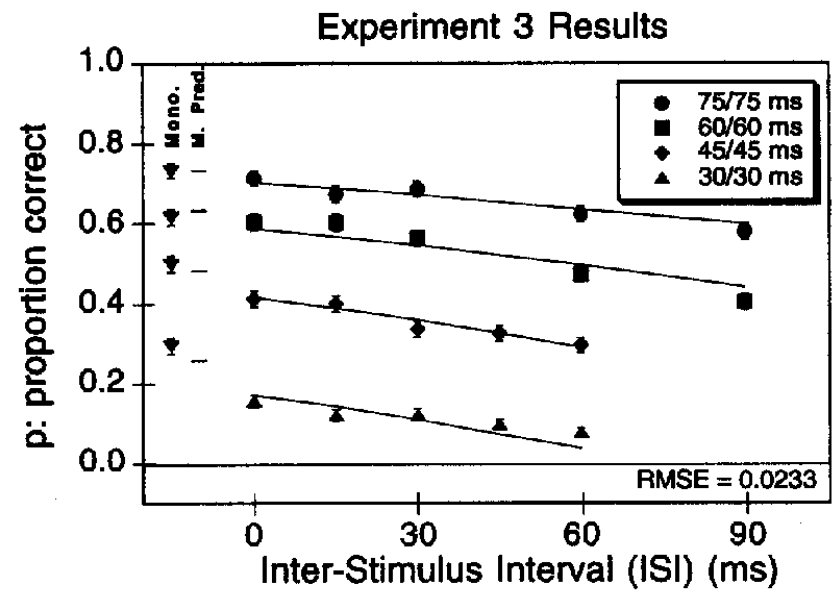

Figure 14. Experiment 3 results: Data are averaged for 3 observers; the solid line shows the best fit of the dual threshold model with a linear combinatorial mechanism. The data show two important features. First, as ISI increases, performance in the dichoptic condition decreases. This finding provides evidence for a postcombinatorial information loss, modeled within the theory as a central sensory threshold. Second, monocular performance for the four monocular stimulus durations (abbreviated Mono. in the figure) is above performance in the 0-ms-ISI dichoptic condition, most markedly at the short-duration $30-\mathrm{ms} / 30-\mathrm{ms}$ and $45-\mathrm{ms} /$ 45-ms presentations. This provides evidence for a precombinatorial information loss, modeled within the theory as a peripheral sensory threshold. The dual threshold model can account for this effect, as indicated by the predicted monocular performance (abbreviated $M$. Pred. in the figure). An analysis of variance $F$ statistic computed as for the data in Figure 9 was significant, $F(20,162)=1.63, p<.05$, which may have resulted from the slight but systematic deviations at the $30-\mathrm{ms} / 30-\mathrm{ms}$ condition.

clear evidence for some form of precombinatorial information loss. Within the context of the dual threshold model, this information loss implies a peripheral sensory threshold.

Taken together, the findings shown in Figure 14 confirm the dual threshold model and reconfirm the existence of a postcombinatorial source of information loss, modeled within the theory as a central sensory threshold.

To summarize the conclusions of Experiment 3, we have again disconfirmed a version of the linear summation model that does not have some form of postcombinatorial information loss, and we have demonstrated that when this postcombinatorial information loss is modeled by a central sensory threshold we can provide good quantitative fits to the data.

\section{General Discussion}

Strong tests of binocular summation come from experiments that vary the temporal onsets of the two presentations of binocular and dichoptic stimuli. We have described a theory that quantitatively accounts for the temporal and ocular summation mechanisms that combine information over time and across the two eyes. Central to this account are two sources of information loss, one prior to and one following the site of binocular combination. We have chosen sensory thresholds as the mechanism of the information loss.
In what follows, we make several remarks about the thresholds in particular, along with general implications of our threshold notions for interpreting binocular superiority. Note that it is not our intent to provide a complete model of binocular combination that accounts for the wide variety of phenomena that exist in the binocular combination literature. Rather, we have embedded a somewhat simplified binocular combination model within our general theory. This model is designed to (a) illustrate the implications of the sensory threshold, (b) examine the mechanisms of binocular combination in conjunction with this threshold, and (c) demonstrate the role of temporal interactions that result from overlapping sensory response functions.

\section{Above-Probability-Summation Performance Does Not Implicate Neural Interaction}

In their review of the binocular summation literature, Blake and Fox (1973; see also Blake et al., 1981) suggested that binocular performance above that predicted via probability summation from monocular performance implies "genuine neural interaction between the eyes, not just probability summation" (Blake et al., 1981, p. 266). They suggested that a modification of the combination rule is required to account for the superiority of binocular performance over the probability summation prediction. We conclude that this need not be the case. We have provided a model that has a linear summation mechanism and an exponential informationacquisition stage. This model, with no postcombinatorial threshold, makes a prediction that is consistent with probability summation. However, this model will also account for the above-probability-summation data from Experiment 1 without altering the nature of the combination mechanism. The existence of a postcombinatorial information loss (modeled by the central threshold) is completely responsible for this prediction.

The logic of this assertion is illustrated in the bottom panel of Figure 5. In the absence of a central threshold, the binocular curve is twice as high as a same-duration monocular curve (with or without a peripheral threshold); correspondingly, the binocular-curve area is twice as great as the monocular-curve area. In the absence of additional mechanisms, this would fulfill a probability summation prediction of $p_{\mathrm{B}}=2 p_{\mathrm{M}}-p_{\mathrm{M}}^{2}$ as a result of the exponential in the information-processing components. However, when a central sensory threshold is introduced, binocular performance rises above what would be predicted from probability summation. This happens because the monocular curve's lower height renders it more vulnerable to a central sensory threshold than a binocular curve. From this perspective, binocular performance is observed to be above what is predicted by probability summation not because observers are doing better in the binocular task, but because they are doing worse in the monocular task. They are doing worse as a direct result of a postcombinatorial information loss, which affects monocular performance more than binocular performance. 


\section{Alternative Ocular-Channel Combination Models}

An explicit assumption of the current linear summation model is that the above-threshold areas from the two monocular channels simply sum to produce the central sensory response function on which performance is based. Although this combination mechanism has support from neuroscience (Ohzawa \& Freeman, 1986) and has been adopted by Legge (1984a, 1984b) and others, alternative mechanisms have been proposed. We now consider in detail a model developed by Cogan (1987; Cogan et al., 1991). A summary of this model follows; a more complete description can be found in Figure 15. We have adapted this model to the current data, and we discuss the extensions to the model that are required to account for the present experimental data. We should point out that we are using Cogan's model to fit

\section{Cogan (1987) Model}

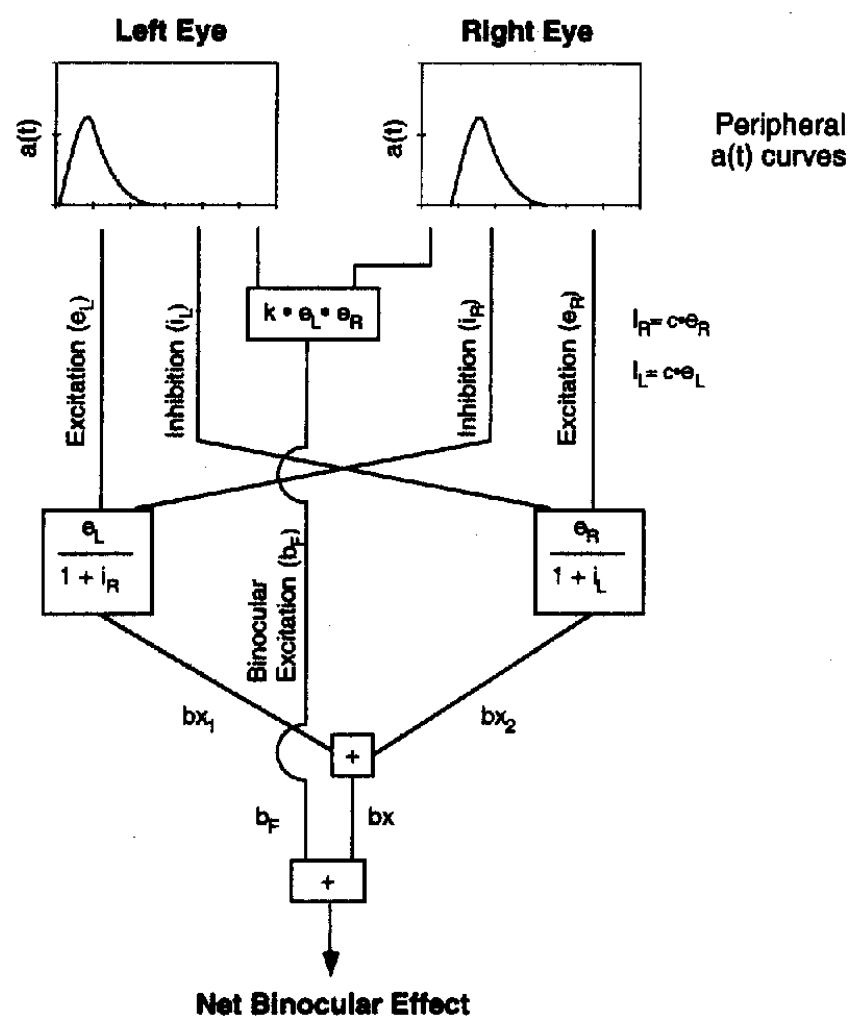

Figure 15. Cogan's (1987) model applied to the data of Experiments 1,2 , and 3 . The height of the peripheral sensory response curves at each point in time is used to construct a net binocular effect curve that is similar to the central sensory response curves in the previous modeling. Each eye contributes an excitatory response that is reduced by an inhibitory response from the other eye $\left(b_{x_{1}}\right.$ and $\left.b_{x_{1}}\right)$. A separate binocular fused channel $\left(b_{\mathrm{F}}\right)$ combines information from the two eyes in a mechanism that allows for facilitation to take place. The either-eye inhibition ( $i_{\mathrm{L}}$ and $i_{\mathrm{R}}$ ) acts as precombination information loss, and the binocular fused channel allows interactions that can act, in principle to imitate a postcombinatorial information loss such as the central sensory response function. However, this model cannot account for the data of Experiments 1, 2 , and 3 without additional assumptions. proportion of correctly recalled digits rather than contrast thresholds, and thus we are not using his model as it was originally developed. In particular, Cogan developed an elegant quantitative formulation that allowed him to calculate, rather than estimate, the parameters of the model. However, this formulation requires threshold data and thus cannot be applied to the present proportion-correct data. As a result, we are adopting only a portion of Cogan's model, that which describes the binocular combination process. We are unable to calculate the values of the model parameters from our performance curves, and thus we treat the model parameters as free parameters.

The Cogan (1987) model assumes that the two eyes perform transduction on incoming stimuli to produce ipsilateral excitation $\left(e_{\mathrm{L}}, e_{\mathrm{R}}\right)$ and contralateral inhibition $\left(i_{\mathrm{L}}, i_{\mathrm{R}}\right)$. The amount of inhibition in one eye is proportional to the excitation in the other eye, such that $i_{\mathrm{R}}=c \cdot e_{\mathrm{L}}$ and $i_{\mathrm{L}}=c \cdot e_{\mathrm{R}}$. The combined either-eye output, $b_{x}$ is given by

$$
b_{x}=\frac{e_{\mathrm{R}}}{\left(1+c \cdot e_{\mathrm{L}}\right)}+\frac{e_{\mathrm{L}}}{\left(1+c \cdot e_{\mathrm{R}}\right)}
$$

A separate, fused channel, $b_{\mathrm{F}}$, is given by

$$
b_{\mathrm{F}}=k \cdot e_{\mathrm{R}} \cdot e_{\mathrm{L}}
$$

and the net binocular effect is simply the sum of $b_{x}$ and $b_{\mathrm{F}}$.

Components of this model share similarities with the dual threshold model presented here. Cogan et al. (1991) developed a linear-filter front end and used this model to fit two-pulse detection thresholds, in which the two pulses could be either the same contrast $(++)$ or opposite contrasts $(+-)$. They systematically varied the SOA and determined relative sensitivities to each type of stimulus. Although they did not obtain quantitative model fits, qualitative model predictions corresponded to the observed data.

Because of the qualitative success of the Cogan (1987) model in accounting for the two-pulse data, we fit the Cogan model to the present data. Before we describe the results of this modeling, some comparisons between the two models are in order.

A major emphasis in the present work is the effects of postcombinatorial information loss on binocular and monocular stimulus representations. An example is the ability of the dual threshold model with a central sensory threshold to account for above-probability-summation binocular performance, even with a linear-summation combination mechanism that would otherwise provide a probability summation prediction. This results from the higher binocular central sensory response function that allows more area to survive a central sensory threshold. The binocular channel $b_{\mathrm{F}}$ in the Cogan (1987) model can in principle have the same effect: If both $e_{\mathrm{L}}$ and $e_{\mathrm{R}}$ are active at once, $b_{\mathrm{F}}$ will be large, giving a larger response and thus better performance. This is qualitatively similar to the two peripheral sensory response curves providing more above-central-threshold area when they co-occur. Thus the parameter $k$ from Cogan's binocular channel is somewhat analogous to the free parameter $\Theta_{c}$ in the dual threshold model, and because we do not have 
threshold data we must estimate $k$ from the data, making it a free parameter as well.

The interocular inhibition from the Cogan (1987) model serves to reduce the response in the opposite monocular channel. This precombinatorial information loss is somewhat analogous to the peripheral thresholds in the dual threshold model. There are important differences in that the peripheral thresholds act only on a single monocular channel, whereas the interocular inhibition crosses channels. However, within the model formulations and the application to our data, precombinatorial information loss is represented by a single free parameter, either $c$ or $\Theta_{\mathrm{p}}$.

Qualitatively, the dual threshold model and the Cogan (1987) model are similar and have the same number of free parameters when applied to proportion-correct data. Given this, it is reasonable to ask whether the Cogan model can fit the data from Experiments 1, 2, and 3. Such fits require an informationprocessing model component to deal with the change from a detection paradigm to a letter identification paradigm.

To assist in model comparison, we simply appended the information-processing mechanisms of the dual threshold model to the output of the Cogan (1987) model (Equation 7). Consistent with the dual threshold model, we assumed that the presentations to each eye engender a peripheral sensory response function corresponding to Cogan's $e_{\mathrm{L}}$ or $e_{\mathrm{R}}$. These two response functions inhibit each other in proportion to their height at each moment in time, according to the free parameter $c$. A separate binocular channel was also created from the peripheral sensory response functions according to the free parameter $k$ via Equation 14. The sum of these two channels gives a central sensory response function. The area of this central sensory response function is used to generate a performance prediction via Equation 7. Table 1 lists the assumptions and predictions for the modified Cogan model.
The Cogan (1987) model could not fit the data as well as the dual threshold model. Parameter values are shown in Table 4. The left panels of Figure 16 show the fits to the averaged data from Experiments 1 and 3 . The data from Experiment 2 could also not be fit but are not shown because the conclusions derived from the data mirror those from Experiment 1 . The reason for these poor fits appears to come from a binocular excitation channel that cannot completely take the place of a central information loss that accounts for both the above-probability-summation binocular performance of Experiment 1 and the decrease in performance with increasing ISI from Experiment 3. When a central nonlinearity such as a central threshold is added to the Cogan model (see Figure 16, right panels), the revised Cogan model gives very good fits in all cases. However, the addition of a central threshold gives the revised model one more free parameter than the dual threshold model.

To summarize, although the data from Experiments 1, 2, and 3 disconfirm the simple version of the Cogan (1987) model, that model gives excellent quantitative fits if a central threshold or central power function is added. Such an addition might be required by the additional complexity of the task: Detection and letter identification might require different spatial and temporal frequencies (Busey, 1994), and letter identification is best thought of as a classification task. The central information loss may result from inefficiencies or noise in the information-processing mechanisms that are not required for simple detection.

\section{Evidence for Temporal Inhibition}

The impulse response function used in the present work (Equation 1) is monophasic and therefore does not allow for temporal inhibition. Busey (1994) found weak evidence for

Table 4

Summary of Best-Fitting Cogan (1987) Model Parameters for Experiments 1-3

\begin{tabular}{|c|c|c|c|c|c|c|c|c|c|c|c|}
\hline \multirow[b]{2}{*}{ Observer } & \multicolumn{5}{|c|}{ Original Cogan model } & \multicolumn{6}{|c|}{ Cogan model with central threshold } \\
\hline & $\tau$ & $c_{s}$ & $c$ & $k$ & RMSE & $\tau$ & $c_{s}$ & $c$ & $k$ & $\theta_{\mathrm{C}}$ & RMSE \\
\hline \multicolumn{12}{|c|}{ Experiment 1} \\
\hline $\begin{array}{l}\text { G.W. } \\
\text { T.B. } \\
\text { T.A.K. } \\
\text { Average }\end{array}$ & $\begin{array}{r}4.62 \\
6.66 \\
31.33 \\
25.19\end{array}$ & $\begin{array}{r}19.51 \\
9.67 \\
12.77 \\
9.91\end{array}$ & $\begin{array}{l}1.98 \times 10^{-6} \\
1.16 \times 10^{4} \\
2.15 \times 10^{18} \\
3.44 \times 10^{6}\end{array}$ & $\begin{array}{r}33.28 \\
82.28 \\
433.54 \\
186.34\end{array}$ & $\begin{array}{l}.0943 \\
.0747 \\
.0634 \\
.0563\end{array}$ & $\begin{array}{r}14.10 \\
6.47 \\
5.66 \\
8.03\end{array}$ & $\begin{array}{l}4.77 \\
1.90 \\
1.44 \\
3.06\end{array}$ & $\begin{array}{l}82.71 \\
48.17 \\
32.05 \\
65.13\end{array}$ & $\begin{array}{l}52.21 \\
20.82 \\
11.36 \\
31.97\end{array}$ & $\begin{array}{l}1.84 \\
2.67 \\
2.50 \\
2.08\end{array}$ & $\begin{array}{l}.0330 \\
.0327 \\
.0370 \\
.0172\end{array}$ \\
\hline \multicolumn{12}{|c|}{ Experiment 2} \\
\hline $\begin{array}{l}\text { G.W. } \\
\text { T.B. } \\
\text { T.K. }\end{array}$ & $\begin{array}{l}2.33 \\
0.65 \\
1.60\end{array}$ & $\begin{array}{r}17.52 \\
11.71 \\
9.62\end{array}$ & $\begin{array}{l}1.39 \times 10^{5} \\
2.27 \times 10^{6} \\
2.48 \times 10^{5}\end{array}$ & $\begin{array}{l}0.00 \\
0.29 \\
0.00\end{array}$ & $\begin{array}{l}.0946 \\
.1107 \\
.1384\end{array}$ & $\begin{array}{l}17.85 \\
23.13 \\
22.22\end{array}$ & $\begin{array}{l}5.28 \\
5.04 \\
3.31\end{array}$ & $\begin{array}{l}33.60 \\
73.70 \\
34.40\end{array}$ & $\begin{array}{r}0.00 \\
57.52 \\
3.59\end{array}$ & $\begin{array}{l}1.32 \\
0.81 \\
0.96\end{array}$ & $\begin{array}{l}.0284 \\
.0413 \\
.0469\end{array}$ \\
\hline \multicolumn{12}{|c|}{ Experiment 3} \\
\hline $\begin{array}{l}\text { T.B. } \\
\text { L.M. } \\
\text { M.B. } \\
\text { Average }\end{array}$ & $\begin{array}{r}28.12 \\
58.84 \\
0.80 \\
31.06\end{array}$ & $\begin{array}{r}15.72 \\
5.02 \\
21.75 \\
12.95\end{array}$ & $\begin{array}{l}5.36 \times 10^{-3} \\
4.24 \times 10^{11} \\
9.41 \times 10^{-6} \\
2.46 \times 10^{-4}\end{array}$ & $\begin{array}{r}49.72 \\
250.40 \\
78.97 \\
19.95\end{array}$ & $\begin{array}{l}.1403 \\
.0786 \\
.0953 \\
.1221\end{array}$ & $\begin{array}{l}30.00 \\
27.08 \\
14.10 \\
20.96\end{array}$ & $\begin{array}{l}6.36 \\
2.71 \\
6.89 \\
5.63\end{array}$ & $\begin{array}{r}4,383.00 \\
649.50 \\
5.50 \\
446.10\end{array}$ & $\begin{array}{r}152.30 \\
118.40 \\
0.00 \\
97.78\end{array}$ & $\begin{array}{l}0.69 \\
0.78 \\
2.15 \\
0.89\end{array}$ & $\begin{array}{l}.0330 \\
.0297 \\
.0376 \\
.0202\end{array}$ \\
\hline
\end{tabular}

Note. Threshold parameter $\Theta_{C}$ is in units of percentage contrast, $\theta$ and $c_{s}$ are in milliseconds, and RMSE (root mean square error) is in units of percentage of correctly recalled digits. Model parameters $c$ and $k$ are from Cogan's (1987) model. $n=9$ for all fits. All fits of the Cogan (1987) model as implemented here were substantially worse than the corresponding dual threshold fits of Table 3 . As a result of this lack of fit, the best-fitting parameter values are often extreme values because the model is stressed to accommodate the data. When a central nonlinearity is added, the values for $c$ and $k$ exhibit much more regularity. 


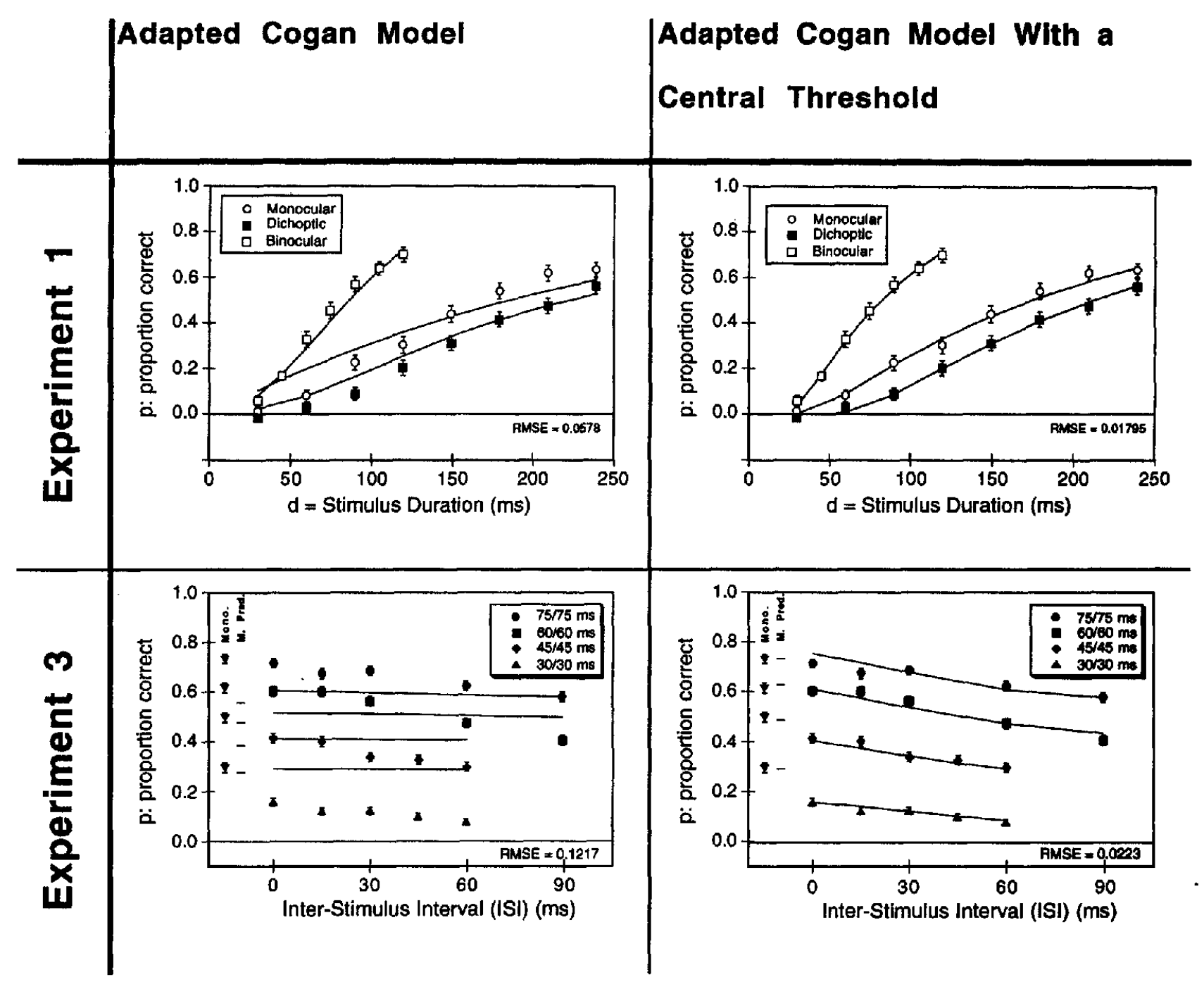

Figure 16. Fits from the Cogan (1987) model adapted to the Experiment 1 and 3 data. Left panels: The adapted Cogan model cannot completely account for the above-probability-summation binocular performance (top left panel) or for the decrease in dichoptic performance with increasing ISI (bottom left panel). Both of these effects require a postcombinatorial source of information loss. When such an information loss source, such as a central sensory threshold, is added, the revised model gives very good fits at the expense of one more free parameter than the dual threshold model. $F$ statistics computed for the fit of the Cogan model with a central threshold to the data of Experiments 1 and 3 were both nonsignificant, $F(18,162)=0.35, p>.05$ and $F(19,162)=1.50, p>.05$, indicating the good fit of the revised model to the data, albeit with one more free parameter than the fits in Figures 9 and 14.

temporal inhibition in digit identification tasks, and when the theory was modified to include temporal inhibition (via a biphasic impulse response function setting $s>0$ in Equation 1) it successfully accounted for the data in these related tasks. However, application of this biphasic model to the present data did not markedly improve the fit over that provided by the monophasic model that we have described (despite two additional free parameters). This failure resulted from the peripheral thresholds, the effects of which tend to eliminate the inhibitory components of the biphasic peripheral sensory response curves. Similarly, a version of the theory that includes temporal inhibition but without peripheral sensory thresholds also did not provide a markedly better fit to any of the three data sets. A version of the Cogan (1987) model with temporal inhibition did not provide fits better than those shown in Figure 16, despite requiring two additional free parameters.

\section{Neuroscience Evidence for a Central Threshold}

Using single-cell recording in cat visual cortex, Ohzawa and Freeman (1986) found overwhelming evidence for 
linear summation in binocular cells. However, a minority of cells exhibited deviations from this linearity in that the response from the cell was maximal when both eyes were equally stimulated but dropped off at a rate faster than would be expected from a linear model when one eye was reduced. This is quite similar to the behavior of the central threshold observed in the current modeling (e.g., see Figure 13). Indeed, Ohzawa and Freeman attributed this nonlinearity to a threshold mechanism that comes into play after the linear binocular summation. One possible mechanism for such a threshold is the threshold for firing on the postsynaptic cell receiving input from both eyes. Weak stimulation to either eye may not exceed this threshold and cause the cell to fire, although weak stimulation to both eyes may result in an action potential on the postsynaptic cell.

\section{Sensory Thresholds: Implications for Cognitive Psychologists}

As noted above, the notion of peripheral and central sensory thresholds is similar to peripheral and central sources of noise. Denis Pelli has demonstrated that under some conditions, either the central or peripheral noise dominates the signal and thus affects performance. He and Manoj Raghavan (Pelli, Raghavan, \& Ahuja, 1995; Raghavan \& Pelli, 1995) demonstrated that the visibility of large, long-duration letters is limited by (central) cortical noise and that the visibility of small, brief-duration letters is limited by (peripheral) photon noise. The important implication for perception researchers is that a stimulus variable such as size can differentially affect two stages of processing (peripheral or central). Thus these two stages become important to model even in experiments that do not use monocular or dichoptic stimuli.

The thresholds as conceptualized above may be thought of as a constant drain on a system that is attempting to process a signal. Consider a metaphor in which the sensory response function represents the speed of some system, say the value of the speedometer in a car. The total amount of information - that is, the area under the sensory response function-is analogous to the odometer reading after some period. Once the threshold is added, however, the metaphor changes to a motorboat traveling upriver. The speed of the current represents the height of the threshold; thus if motorboat speed does not exceed the current speed, then no upriver progress will be made.

\section{Conclusions}

We have extended a previously proposed theory of visual information processing to the domain of binocular combination. Application of this extended theory to data from three experiments helps identify the sources of information loss, both pre- and postcombination. Finally, we have confirmed a version of the extended theory that combines both pre- and postcombinatorial sources of information loss with a linearsummation combination mechanism and an independent sampling information-acquisition mechanism. This application demonstrates that the question of the combination mechanisms involved in binocular combination is intimately tied to the question of pre- and postcombination sources of information loss: One cannot simply look at monocular and binocular data and infer the combinatorial mechanisms without also considering the potential for something like peripheral and central sensory response thresholds. In particular, the existence of a central threshold implies that binocular performance above that predicted by probability summation on the basis of monocular data need not implicate an above-linear-summation combination mechanism. A linear summation mechanism, an independent sampling information-acquisition mechanism, and a central threshold will provide an equivalent account.

The linear-filter model components also allow a good quantitative account of the degree to which the relationship between monocular and binocular performance changes as the asynchrony between the two pulses of the binocular stimulus changes. Such modeling reveals not only under what conditions the two monocular channels influence each other but also how they are affected by the postcombinatorial information loss. Finally, we have demonstrated that such quantitative fits are important when comparing two models that have similar structure and components and thus give qualitatively similar results.

\section{References}

Ames, C., \& Palmer, J. (1992). Macintosh experimental software [Unpublished computer code]. Seattle, WA: Authors.

Blake, R., \& Fox, R. (1973). The psychophysical inquiry into binocular summation. Perception \& Psychophysics, 14, 161-185.

Blake, R., Sloane, M., \& Fox, R. (1981). Further developments in binocular summation. Penception \& Psychophysics, 30, 266-276.

Burr, D., \& Morrone, M. (1996). Temporal impulse response functions for luminance and colour during saccades. Vision Research, 36, 2069-2078.

Busey, T. A. (1994). Temporal inhibition in two-pulse character detection and identification tasks. Unpublished doctoral dissertation, University of Washington, Seattle.

Busey, T. A., \& Loftus, G. R. (1994). Sensory and cognitive components of visual information acquisition. Psychological Review, 10I, 446-469.

Cogan, A. (1987). Human binocular interaction: Towards a neural model. Vision Research, 27, 2125-2139.

Cogan, A., Clarke, M., Chan, H., \& Rossi, A. (1991). Two-pulse monocular and binocular interactions at the differential luminance threshold. Vision Research, 30, 1617-1630.

Eriksen, C. W., \& Greenspon, T. S. (1968). Binocular summation over time in the perception of form at brief durations. Journal of Experimental Psychology, 76, 331-336.

Eriksen, C. W., Greenspon, T. S., Lappin, J., \& Carison, W. A. (1966). Binocular summation in the perception of form at brief durations. Perception \& Psychophysics, 1, 415-419.

Green, D., \& Swets, J. (1974). Signal detection theory and psychophysics. Huntington, NY: Robert E. Krieger.

Julesz, B. (1971). Foundations of Cyclopean perception. Chicago: University of Chicago Press.

Legge, G. (1984a). Binocular contrast summation-I. Detection and discrimination. Vision Research, 24, 373-383.

Legge, G. (1984b). Binocular contrast summation-II. Quadratic summation. Vision Research, 24, 385-394.

Loftus, G. R., Busey, T. A., \& Senders, J. (1993). Providing a 
sensory basis for models of visual information acquisition. Perception \& Psychophysics, 54, 535-554.

Loftus, G. R., Duncan, J., \& Gehrig, P. (1992). On the time course of perceptual information that results from a brief visual presentation. Journal of Experimental Psychology: Human Perception and Performance, 18, 530-549.

Loftus, G. R., \& Irwin, D. E. (in press). On the relations among different measures of visible and informational persistence. Cognitive Psychology.

Loftus, G. R., \& McLean, J. (1998). A front end to a theory of picture recognition. Manuscript in preparation.

Loftus, G. R., \& Ruthruff, E. R. (1994). A theory of visual information acquisition and visual memory with special application to intensity-duration tradeoffs. Journal of Experimental Psychology: Human Penception and Performance, 20, 33-50.

Massaro, D. W. (1970). Perceptual processes and forgetting in memory tasks. Psychological Review, 77, 557-567.

Matin, L. (1962). Binocular summation at the absolute threshold of peripheral vision. Joumal of the Optical Society of America, 52, 1276-1286.

Ohzawa, I., \& Freeman, R. D. (1986). The binocular organization of simple cells in the cat's visual cortex. Journal of Neurophysiology, 56, 221-242.

Pelli, D. G., Raghavan, M., \& Ahuja, S. (1995). The noises that limit visual perception. In Proceedings of the 1995 Association for Research in Vision and Ophthalmology Conference, 36, S851.
Raghavan, M., \& Pelli, D. G. (1995). Psychophysical evidence for cortical noise. In Proceedings of the 1995 Association for Research in Vision and Ophthalmology Conference, 36, \$905.

Rumelhart, D. E. (1970). A multicomponent theory of the perception of briefly exposed visual displays. Journal of Mathematical Psychology, 7, 191-218.

Shibuya, H., \& Bundesen, C. (1988). Visual selection from multielement displays: Measuring and modeling effects of exposure duration. Journal of Experimental Psychology: Human Perception and Performance, 14, 591-600.

Sperling, G., \& Melchner, M. J. (1978). Visual search, visual attention, and the attention operating characteristics. In J. Requin (Ed.), Attention and performance VII. Hillsdale, NJ: Erlbaum.

Sperling, G., \& Sondhi, M. M. (1968). Model for visual luminance discrimination and flicker detection. Journal of the Optical Society of America, 58, 1133-1145.

Townsend, J. T. (1981). Some characteristics of visual whole report behavior. Acta Psychologica, 47, 149-173.

Watson, A. (1979). Probability summation over time. Vision Research, 19, 515-522.

Watson, A. B. (1986). Temporal sensitivity. In K. R. Boff, L. Kaufman, \& J. P. Thomas (Eds.), Handbook of perception and human performance (Vol. 1, pp. 6-1 to 6-43). New York: Wiley.

Westendorf, D., Blake, R., \& Fox, R. (1972). Binocular summation of equal energy flashes of unequal duration. Perception \& Psychophysics, 12, 445-448.

\section{Appendix A}

\section{Equations for All Model Fits}

Parameter fitting was accomplished with a simplex gradient descent method. To avoid the possibilities of local minima, we computed the best-fitting parameters multiple times, each time from a different starting location in parameter space. The vast majority of parameter searches terminated at the same ending parameters for a given model fit.

All model fits derive the peripheral sensory response functions, $a_{\mathrm{p}}(t)$, via the convolution of the stimulus wave form with the impulse-response function of the linear filter:

$$
a_{\mathrm{p}}(t)=f(t) * g(t)
$$

where $g(t)$ is the gamma function from Equation 1. Note that for monocular and dichoptic stimuli, the left-eye and right-eye $f(t)$ functions will differ according to which eye is stimulated (or stimulated first in the dichoptic case).

We develop two forms of the nonlinearity. The first is a hard threshold that assumes that information acquisition does not begin until the sensory response function exceeds the sensory threshold. The second is a soft threshold that raises the sensory response function to a power in line with Watson's (1979) probability-summation-in-time theory.

\section{Predictions for the Dual Threshold Model}

A peripheral threshold, $\Theta_{\mathrm{p}}$, is assessed on the peripheral $a(t)$ wave form for the hard threshold version of the theory,

$$
a_{\Theta, p}(t)=\begin{array}{ll}
a_{\mathrm{p}}(t)-\Theta_{\mathrm{p}} & \left(a(t)>\Theta_{\mathrm{p}}\right) \\
0 & \left(a(t) \leq \Theta_{\mathrm{p}}\right)
\end{array}
$$

or a power function is applied to the peripheral $a(t)$ wave form:

$$
a_{\beta, p}(t)=\left[a_{\mathrm{p}}(t)\right]^{\beta}
$$

The two peripheral sensory response functions sum to create the central sensory response function, $a_{\mathrm{c}}(t)$ :

$$
a_{c}(t)=a_{\Theta, \mathrm{L}}(t)+a_{\Theta, \mathrm{R}}(t)
$$

or

$$
a_{\mathrm{c}}(t)=a_{\beta, \mathrm{L}}(t)+a_{\beta, \mathrm{R}}(t)
$$

for the power-function version of the theory.

In the dual threshold model, a central threshold is assessed to create $a_{\theta, c}(t)$ for the hard threshold version of the theory,

$$
a_{\theta, c}(t)=\begin{array}{ll}
a_{c}(t)-\Theta_{c} & \left(a_{c}(t)>\Theta_{c}\right) \\
0 & \left(a_{c}(t) \leq \Theta_{c}\right)
\end{array}
$$

or a power function is applied to the central $a(t)$ wave form:

$$
a_{\beta, c}(t)=\left[a_{c}(t)\right]^{\beta}
$$

The area under the $a_{\Theta, c}(t)$ function is converted to performance 
via the following formula:

$$
p=1.0-e^{\left.-A_{\theta, c}(0)\right\rangle c_{s}} .
$$

For the power-function version of the theory, performance is a function of the area under the $a_{\beta, c}(t)$ wave form:

$$
p=1.0-\mathrm{e}^{-A_{R, c}(\infty) / c_{s}}
$$

\section{Predictions for the Cogan Model}

Using the notation of the Busey and Loftus (1994) model, the Cogan (1987) model is formulated as follows:

$$
b_{x}(t)=\frac{a_{\mathrm{R}}(t)}{\left[1+c \cdot a_{\mathrm{L}}(t)\right]}+\frac{a_{\mathrm{L}}(t)}{\left[1+c \cdot a_{\mathrm{R}}(t)\right]}
$$

and

$$
b_{\mathrm{F}}(t)=k \cdot a_{\mathrm{R}}(t) \cdot a_{\mathrm{L}}(t)
$$

where $a_{\mathrm{R}}(t)$ and $a_{\mathrm{L}}(t)$ represent $e_{\mathrm{R}}$ and $e_{\mathrm{L}}$, respectively. The central sensory response function $a_{\mathrm{c}}(t)$ is computed as follows:

$$
a_{\mathrm{c}}(t)=b_{\mathrm{x}}(t)+b_{\mathrm{F}}(t) .
$$

If a central threshold is applied after the point of combination, this formulation is added to create $a_{\theta, c}(t)$ for the hand threshold version,

$$
a_{\theta, c}(t)=\begin{array}{ll}
a_{c}(t)-\Theta_{c} & \left(a_{c}(t)>\Theta_{c}\right) \\
0 & \left(a_{c}(t) \leq \Theta_{c}\right)
\end{array}
$$

or a power function is applied to the central $a(t)$ wave form:

$$
a_{\beta, c}(t)=\left[a_{\mathrm{c}}(t)\right]^{\beta} .
$$

In all three cases, performance is converted from the area under the central sensory response function via Equation 7 :

$$
p=1.0-e^{\left.-A_{c}(\infty)\right) c_{s}}
$$

where $A_{c}(\infty)$ is replaced by $A_{\theta, c}(\infty)$ when a central threshold is added or by $A_{\beta, c}(\infty)$ when a central power function is added. 


\section{Appendix B}

\section{Glossary}

\begin{tabular}{|c|c|c|c|}
\hline Term & Definition & Units & $\begin{array}{l}\text { Depends } \\
\text { on }\end{array}$ \\
\hline \multicolumn{4}{|c|}{ Physical attributes } \\
\hline$d$ & Stimulus duration & Milliseconds & \\
\hline$t$ & Time since stimulus onset & Milliseconds & \\
\hline$F$ & Stimulus contrast & Percentage (contrast) & \\
\hline \multicolumn{4}{|c|}{ Performance measures } \\
\hline$p$ & $\begin{array}{l}\text { Proportion of digits recalled (corrected for } 10 \% \text { guessing } \\
\text { probability) }\end{array}$ & Proportion & \\
\hline \multicolumn{4}{|c|}{ Linear-systems parameters } \\
\hline $\begin{array}{l}t \\
n \\
\Theta \\
\Theta_{P} \\
\Theta_{C} \\
\beta_{p} \\
\beta_{C}\end{array}$ & $\begin{array}{l}\text { Decay constant of the impulse-response function } \\
\text { Number of stages in the impulse-response function } \\
\text { Sensory threshold } \\
\text { Peripheral sensory threshold } \\
\text { Central sensory threshold } \\
\text { Peripheral power function } \\
\text { Central power function }\end{array}$ & $\begin{array}{l}\text { Milliseconds } \\
\text { Integer } \\
\text { Percentage (contrast) } \\
\text { Percentage (contrast) } \\
\text { Percentage (contrast) } \\
\text { Percentage (contrast) } \\
\text { Percentage (contrast) }\end{array}$ & \\
\hline \multicolumn{4}{|c|}{ Linear-systems functions } \\
\hline$f(t)$ & Stimulus-contrast function & Percentage (contrast) & \\
\hline$F(t)$ & Integral from 0 to $t$ of $f(t)$ & Milliseconds & \\
\hline$F(\infty)$ & Total area under $f(t)$ & Milliseconds & \\
\hline$g(t)$ & Impulse-response function & Percentage (contrast) & $t, n$ \\
\hline$G(t)$ & Integral from 0 to $t$ of $g(t)$ & Milliseconds & $t, n$ \\
\hline$G(\infty)$ & Total area under $g(t)=1.0$ & Milliseconds & $t, n$ \\
\hline$a(t)$ & Sensory response function & Percentage (contrast) & $f(t), t, n$ \\
\hline$A(t)$ & Integral from 0 to $t$ of $a(t)$ & Milliseconds & $f(t), t, n$ \\
\hline$A(\infty)$ & Total area under $a(t)$ & Milliseconds & $f(t), t, n$ \\
\hline $\begin{array}{l}a_{\Theta}(t)= \\
a(t)-0\end{array}$ & Above-threshold sensory response function & Percentage (contrast) & $f(t), t, n, \Theta$ \\
\hline$a_{\theta_{\mathrm{p}}}(t)$ & Above-peripheral-threshold sensory response function & Percentage (contrast) & $f(t), t, n, \Theta_{\mathrm{p}}$ \\
\hline$a_{\theta}(t)$ & Above-central-threshold sensory response function & Percentage (contrast) & $f(t), t, n, \Theta_{\mathrm{C}}$ \\
\hline $\begin{array}{l}A_{\Theta}(t) \\
A_{\Theta}(\infty)\end{array}$ & $\begin{array}{l}\text { Integral from } 0 \text { to } t \text { of } a_{\theta}(t) \\
\text { Total area under } a_{\Theta}(t)\end{array}$ & $\begin{array}{l}\text { Milliseconds } \\
\text { Milliseconds }\end{array}$ & $\begin{array}{l}f(t), t, n, \Theta \\
f(t), t, n, \Theta\end{array}$ \\
\hline \multicolumn{4}{|c|}{ Extraction-rate parameter } \\
\hline$c_{s}$ & Reciprocal of raw sampling rate & Milliseconds & \\
\hline \multicolumn{4}{|c|}{ Extraction-rate and performance functions } \\
\hline$r(t)$ & Information-extraction rate function & Milliseconds $^{-1}$ & $f(t), \tau, n, \Theta, c_{s}$ \\
\hline$I(t)$ & Extracted-information function & Proportion & $f(t), \tau, n, \theta, c_{s}$ \\
\hline$I(\infty)$ & Total extracted information & Proportion & $f(t), \tau, n, \Theta, c_{s}$ \\
\hline \multicolumn{4}{|c|}{ Modified Cogan (1987) model parameters and functions } \\
\hline $\begin{array}{l}c \\
k \\
b_{x}(t) \\
b_{\mathrm{F}}(t) \\
a_{\mathrm{C}}(t)\end{array}$ & $\begin{array}{l}\text { Degree of inhibition from contralateral eye } \\
\text { Amount of fused binocular energy } \\
\text { Total either-eye output } \\
\text { Total fused output } \\
\text { Net binocular effect }\left[b_{x}(t)+b_{F}(t)\right]\end{array}$ & $\begin{array}{l}\text { Proportion } \\
\text { Proportion } \\
\text { Percentage (contrast) } \\
\text { Percentage (contrast) } \\
\text { Percentage (contrast) }\end{array}$ & $\begin{array}{l}f(t), t, n, c \\
f(t), t, n, c \\
f(t), t, n, c, k\end{array}$ \\
\hline
\end{tabular}

Received November 26, 1996 Revision received May 12, 1997 Accepted June 30, 1997 